\title{
Internal states of model isotropic granular packings. I. Assembling process, geometry and contact networks.
}

\author{
Ivana Agnolin* and Jean-Noël Rouxiّ \\ Laboratoire des Matériaux et des Structures du Génie Civil ${ }^{\ddagger}$, Institut Navier, \\ 2 allée Kepler, Cité Descartes, 77420 Champs-sur-Marne, France
}

(Dated: October 22, 2018)

\begin{abstract}
This is the first paper of a series of three, in which we report on numerical simulation studies of geometric and mechanical properties of static assemblies of spherical beads under an isotropic pressure. The influence of various assembling processes on packing microstructures is investigated. It is accurately checked that frictionless systems assemble in the unique random close packing (RCP) state in the low pressure limit if the compression process is fast enough, higher solid fractions corresponding to more ordered configurations with traces of crystallization. Specific properties directly related to isostaticity of the force-carrying structure in the rigid limit are discussed. With frictional grains, different preparation procedures result in quite different inner structures that cannot be classified by the sole density. If partly or completely lubricated they will assemble like frictionless ones, approaching the RCP solid fraction $\Phi_{\mathrm{RCP}} \simeq 0.639$ with a high coordination number: $z^{*} \simeq 6$ on the force-carrying backbone. If compressed with a realistic coefficient of friction $\mu=0.3$ packings stabilize in a loose state with $\Phi \simeq 0.593$ and $z^{*} \simeq 4.5$. And, more surprisingly, an idealized "vibration" procedure, which maintains an agitated, collisional régime up to high densities results in equally small values of $z^{*}$ while $\Phi$ is close to the maximum value $\Phi_{\mathrm{RCP}}$. Low coordination packings have a large proportion $(>10 \%)$ of rattlers - grains carrying no force - the effect of which should be accounted for on studying position correlations, and also contain a small proportion of localized "floppy modes" associated with divalent grains. Low pressure states of frictional packings retain a finite level of force indeterminacy even when assembled with the slowest compression rates simulated, except in the case when the friction coefficient tends to infinity. Different microstructures are characterized in terms of near neighbor correlations on various scales, and some comparisons with available laboratory data are reported, although values of contact coordination numbers apparently remain experimentally inaccessible.
\end{abstract}

PACS numbers: 45.70.-n, 83.80.Fg, 46.65.+g, 62.20.Fe

\section{INTRODUCTION}

\section{A. Context and motivations}

The mechanical properties of solidlike granular packings and their microscopic, grain-level origins are an active field of research in material science and condensedmatter physics [1, 2, 3]. Motivations are practical, originated in soil mechanics and material processing, as well as theoretical, as general approaches to the rheology of different physical systems made of particle assemblies out of thermal equilibrium [4] are attempted.

The packing of equal-sized spherical balls is a simple model for which there is a long tradition of geometric characterization studies. Packings are usually classified according to their density or solid volume fraction $\Phi$, and the frequency of occurrence of some local patterns. Direct observations of packing microstructure is difficult, although it has recently benefitted from powerful imag-

\footnotetext{
* Present address: Geoforschungszentrum, Haus D, Telegrafenberg, D-14473 Potsdam, Germany

$\ddagger$ LMSGC is a joint laboratory depending on Laboratoire Central des Ponts et Chaussées, École Nationale des Ponts et Chaussées and Centre National de la Recherche Scientifique

${ }^{\dagger}$ Electronic address: jean-noel.roux@lcpc.fr
}

ing techniques [ [5, 6, 7]. The concept of random close packing (RCP), is often invoked [8, 9], although some authors criticized it as ill-defined [10]. It corresponds to the common observation that bead packings without any trace of crystalline order do not exceed a maximum density, $\Phi_{R C P}$, slightly below $0.64[8]$.

Mechanical studies in the laboratory have been performed on granular materials for decades in the realm of soil mechanics, and the importance of packing fraction $\Phi$ on the rheological behavior has long been recognized [1, 11, 12, 13]. The anisotropy of the packing microstructure, due to the assembling process, has also been investigated [14, 15], and shown to influence the stress-strain behavior of test samples [16], as well as the stress field and the response to perturbations of gravitystabilized sandpiles or granular layers [17].

Discrete numerical simulation [18] proved a valuable tool to investigate the internal state of packings, as it is able to reproduce mechanical behaviors, and to identify relevant variables other than $\Phi$, such as coordination number and fabric (or distribution of contact orientations) [19, 20, 21, 22, 23]. In the case of sphere packings, simulations have been used to characterize the geometry of gravity-deposited systems [24, 25] or oedometrically compressed ones [26], to investigate the quasistatic, hysteretic stress-strain dependence in solid packings [27, 28], and their pressure-dependent elastic moduli in a com- 
pression experiment [29, 30].

However, in spite of recent progress, quite a few basic questions remain unresolved. It is not obvious how closely the samples used in numerical simulations actually resemble laboratory ones, for which density is often the only available state parameter. Both simulations and experiments resort to certain preparation procedures to assemble granular packings, which, although their influence is recognized as important, are seldom studied, or even specified. One method to produce dense packings in simulations is to set the coefficient of intergranular friction to zero [26, 27] or to a low value [28] in the assembling stage, while a granular gas gets compacted and equilibrated under pressure. On keeping frictionless contacts, this results, in the limit of low confining stress, in dense systems with rather specific properties 30, 31], related to isostaticity and potential energy minimization [32]. Examples of traditional procedures in soil mechanics are rain deposition under gravity, also known as air pluviation (which produces homogeneous states if grain flow rate and height of free fall [33] are maintained constant) and layerwise deposition and dry or moist tamping. Those two methods were observed to produce, in the case of loose sands, different structures for the same packing density [34]. Densely packed particle assemblies can also be obtained in the laboratory by vibration, or application of repeated "taps" 35, 36] to a loose deposit. How close are dense experimental sphere packings to model configurations obtained on simulating frictionless particles ? How do micromechanical parameters influence the packing structure? Is the low pressure limit singular in laboratory grain packings and in what sense?

\section{B. Outline of the present study}

The present paper provides some answers to such questions, from numerical simulations in the simple case of isotropically assembled and compressed homogeneous packings of spherical particles. It is the first one of a series of three, and deals with the geometric characterization of low pressure isotropic states assembled by different procedures, both without and with intergranular friction. The other two, hereafter referred to as papers II 37] and III [38], respectively investigate the effects of compressions and pressure cycles, and the elastic response of the different numerical packings, with comparisons to experimental results. Although mechanical aspects are hardly dealt with in the present paper, we insist that geometry and mechanics are strongly and mutually related. We focus here on the variability of the coordination numbers, which will prove important for mechanical response properties of granular packings, and show that equilibrated packs of identical beads can have a relatively large numbers of "rattler" grains, which do not participate in force transmission. We investigate the dependence of initial states on the assembling procedure, both with and without friction. We study the effects of procedures designed to produce dense states (close to RCP), and we characterize the geometry of such states on different scales.

It should be emphasized that we do not claim here to mimic experimental assembling procedures very closely. Rather, we investigate the results of several preparation methods, which are computationnally convenient, maintain isotropy, and produce equilibrated samples with rather different characteristics. Those methods nevertheless share some important features with laboratory procedures, and we shall argue that the resulting states are plausible models for experimental samples.

The numerical model and the simulation procedures (geometric and mechanical parameters, contact law, boundary conditions) are presented in Section II, where some basic definitions and mechanical properties pertaining to granular packs are also presented or recalled. Part III discusses the properties of frictionless packings, and introduces several characterization approaches used in the general case as well. Section IV then describes different assembling procedures of frictional packings and the resulting microstructures. Section $\mathrm{V}$ discusses perspectives to the present study, some of which are pursued in papers II and III of the series. Appendices deal with technical issues, and also present a more detailed comparison with some experimental data.

This being a long paper, it might be helpful to specify which parts can be read independently. On first going through the paper, the reader might skip Section IIID. dealing with a rather specific issue. The properties stated or recalled in Section [I C are used to discuss stability issues and isostatic values of coordination numbers, but they can also be overlooked in a first approach. Finally, Section IV can be read independently from Section III. apart from the explanations about equilibrium conditions (in paragraph III B 2) and the treatment of rattlers (paragraph [IIE 1). Sections III and IV both have conclusive subsections which summarize the essential results.

\section{MODEL, NUMERICAL PROCEDURES, BASIC DEFINITIONS}

\section{A. Intergranular forces}

We consider spherical beads of diameter $a$ (the value of which, as we ignore gravity, will prove irrelevant), interacting in their contacts by the Hertz law, relating the normal force $N$ to the elastic normal deflection $h$ as :

$$
N=\frac{\tilde{E} \sqrt{a}}{3} h^{3 / 2} .
$$

In Eqn. 1] we introduced the notation

$$
\tilde{E}=\frac{E}{1-\nu^{2}},
$$


$E$ is the Young modulus of the beads, and $\nu$ the Poisson ratio. For spheres, $h$, the elastic deflection of the contact, is simply the distance of approach of the centers beyond the first contact. The normal stiffness $K_{N}$ of the contact is defined as the rate of change of the force with normal displacement:

$$
K_{N}=\frac{d N}{d h}=\frac{\tilde{E} \sqrt{a}}{2} h^{1 / 2}=\frac{3^{1 / 3}}{2} \tilde{E}^{2 / 3} a^{1 / 3} N^{1 / 3}
$$

Although many geometric features of particle packings do not depend on the details of the model for contact elasticity, and could be observed as well with a simpler, linear unilateral elastic model, it is necessary to implement suitable non-linear contact models to deal with the mechanical properties in papers II and III [37, 38]. Tangential elasticity and friction in contacts are appropriately described by the Cattaneo-Mindlin-Deresiewicz laws [39], which we implement in a simplified form, as used e.g., in refs. 29, 41]: the tangential stiffness $K_{T}$ relating, in the elastic regime, the increment of tangential reaction $d \mathbf{T}$ to the relative tangential displacement increment $d \mathbf{u}_{T}$ is a function of $h$ (or $N$ ) alone (i.e., it is kept constant, equal to its value for $\mathbf{T}=0)$ :

$$
\begin{aligned}
d \mathbf{T} & =K_{T} d \mathbf{u}_{T}, \quad \text { with } \\
K_{T} & =\frac{2-2 \nu}{2-\nu} K_{N}=\frac{1-\nu}{2-\nu} \tilde{E} \sqrt{a} h^{1 / 2}
\end{aligned}
$$

To enforce the Coulomb condition with friction coefficient $\mu, \mathbf{T}$ has to be projected back onto the circle of radius $\mu N$ in the tangential plane whenever the increment given by eqn. 3 would cause its magnitude to exceed this limit. Moreover, when $N$ decreases to $N-\delta N$, T is scaled down to the value it would have had if $N$ had constantly been equal to $N-\delta N$ in the past. It is not scaled up when $N$ increases. Such a procedure, suggested e.g., in [40], avoids spurious increases of elastic energy for certain loading histories. More details are given in Appendix $\mathrm{A}$

Finally, tangential contact forces have to follow the material motion. Their magnitudes are assumed here not to be affected by rolling (i.e., rotation about a tangential axis) or pivoting (i.e., rotation about the normal axis), while their direction rotates with the normal vector due to rolling, and spins around it with the average spinning rate of the two spheres (to ensure objectivity). The corresponding equations are given in Appendix B.

In addition to the contact forces specified above, we introduce viscous ones, which oppose the normal relative displacements (we use the convention that positive normal forces are repulsive):

$$
N^{v}=\alpha(h) \dot{h}
$$

The damping coefficient $\alpha$ depends on $h$, and we choose its value as a fixed fraction $\zeta$ of the critical damping coefficient of the normal (linear) spring of stiffness $K_{N}(h)$ (as given by (2) ) joining two beads of mass $m$ :

$$
\alpha(h)=\zeta \sqrt{2 m K_{N}(h)} .
$$

From (2), $\alpha$ is thus proportional to $h^{1 / 4}$, or to $N^{1 / 6}$. The same damping law was used in [41]. Admittedly, the dissipation given by (4)-(5) has little physical justification, and is rather motivated by computational convenience. We shall therefore assess the influence of $\zeta$ on the numerical results. The present study being focussed on statics, we generally use a strong dissipation, $\zeta=0.98$, to approach equilibrium faster. This particular value is admittedly rather arbitrary: the initial motivation for choosing $\zeta<1$ is the computational inefficiency of overdamped contacts with $\zeta>1$ in the case of linear contact elasticity. Yet we did not check whether values of 1 or even higher would cause any problem with Hertzian contacts. In the linear case, the restitution coefficient in a binary collision varies as a very fastly decreasing function of $\zeta$, and changes of $\zeta$ in the range between 0.7 and 1 have virtually no detectable effect.

We do not introduce any tangential viscous force, and impose the Coulomb inequality to elastic force components only. We choose the elastic parameters $E=70 \mathrm{GPa}$ and $\nu=0.3$, suitable for glass beads, and the friction coefficient is attributed a moderate, plausible value $\mu=0.3$. These choices are motivated by comparisons to experimental measurements of elastic moduli, to be carried out in paper III [38].

\section{B. Boundary conditions and stress control}

The numerical results presented below were obtained on samples of $n=4000$ beads, enclosed in a cubic or parallelipipedic cell with periodic boundary conditions.

It is often in our opinion more convenient to use pressure (or stress) than density (or strain) as a control parameter (a point we discuss below in Section IIII). We therefore use a stress-controlled procedure in our simulations, which is adapted from the Parrinello-Rahman molecular dynamics (MD) scheme [42]. The simulation cell has a rectangular parallelipipedic shape with lengths $L_{\alpha}$ parallel to coordinate axes $\alpha(1 \leq \alpha \leq 3)$. $L_{\alpha}$ values might vary, so that the system has $6 N+3$ configurational degrees of freedom, which are the positions and orientations of the $N$ particles and lengths $L_{\alpha} . \quad \Omega=L_{1} L_{2} L_{3}$ denotes the sample volume. We seek equilibrium states with set values $\left(\Sigma_{\alpha}\right)_{1 \leq \alpha \leq 3}$ of all three principal stresses $\sigma_{\alpha \alpha}$. We use the convention that compressive stresses are positive.

It is convenient to write position vectors $\mathbf{r}_{i}$, defining a square $3 \times 3$ matrix with $L_{\alpha}$ 's on the diagonal, as

$$
(1 \leq i \leq N) \quad \mathbf{r}_{i}=\underline{\underline{\mathbf{L}}} \cdot \mathbf{s}_{i},
$$

$\mathbf{s}_{i}$ denoting corresponding vectors in a cubic box of unit edge length. In addition to particle angular and linear velocities, which read

$$
(1 \leq i \leq N) \quad \mathbf{v}_{i}=\underline{\underline{\mathbf{L}}} \cdot \dot{\mathbf{s}}_{i}+\underline{\underline{\mathbf{L}}} \cdot \mathbf{s}_{i},
$$

one should evaluate time derivatives $\dot{L}_{i}$. Equations of motion are written for particles in the standard form, 
i.e., $\left(\mathbf{F}_{i}\right.$ denoting the total force exerted on grain $\left.i\right)$

$$
(1 \leq i \leq N) \quad m_{i} \ddot{\mathbf{s}}_{i}=\underline{\underline{L}}^{-1} \cdot \mathbf{F}_{i}
$$

and the usual equation for angular momentum. Meanwhile, lengths $\dot{L}_{\alpha}$ satisfy the following equation of motion, in which $\mathbf{r}_{i j}$ is the vector joining the center of $i$ to the center of $j$, subject to the usual nearest image convention of periodic boundary conditions:

$$
\begin{aligned}
M \ddot{L}_{\alpha} & =\frac{1}{L_{\alpha}}\left[L_{\alpha}^{2} \sum_{i} m_{i}\left(\dot{s}_{i}^{(\alpha)}\right)^{2}+\sum_{i<j} F_{i j}^{(\alpha)} r_{i j}^{(\alpha)}\right] \\
& -\frac{\Omega}{L_{\alpha}} \Sigma_{\alpha} .
\end{aligned}
$$

Within square brackets on the right-hand side of Eqn. 7. one recognizes the familiar formula [19, 43, 44] for $\Omega \sigma_{\alpha \alpha}$, $\underline{\underline{\sigma}}$ being the average stress in the sample:

$$
\sigma_{\alpha \beta}=\frac{1}{\Omega}\left[\sum_{i} m_{i} v_{i}^{\alpha} v_{i}^{\beta}+\sum_{i<j} F_{i j}^{(\alpha)} r_{i j}^{(\alpha)}\right]
$$

All three diagonal stress components should thus equate the prescribed values $\Sigma_{\alpha}$ at equilibrium. The acceleration term will cause the cell to expand in the corresponding direction if the stress is too high, and to shrink if it is too low. Eqn. 7 involves a generalized mass $M$ associated with the changes of shape of the simulation cell. $M$ is set to a value of the order of the total mass of all particles in the sample. This choice was observed to result in collective degrees of freedom $L_{\alpha}$ approaching their equilibrium values under prescribed stress $\underline{\boldsymbol{\Sigma}}$ somewhat more slowly (but not exceedingly so) than (rescaled) positions s.

The original Parrinello-Rahman method was designed for conservative molecular systems, in such a way that the set of equations is cast in Lagrangian form. This implies in particular additional terms in (6), involving $\underline{\underline{\mathbf{L}}}$. Such terms were observed to have a negligible influence on our calculations and were consequently omitted. Granular materials are dissipative, and energy conservation is not an issue (except for some elastic properties, see paper III [38]). Further discussion of the stress-controlled method is provided in Appendix C.

Equations (6) and (7), with global degrees of freedom $L_{\alpha}$ slower than particle positions, lead to dynamics similar to those of a commonly used procedure in granular simulation [45]. This method consists in repeatedly changing the dimensions of the cell by very small amounts, then computing the motion of the grains for some interval of time. A "servo mechanism" can be used to impose stresses rather than strains [30]. Our approach might represent a simplification, as it avoids such a two-stage procedure. It should be kept in mind that we restricted our use of equation (7) to situations when changes in the dimensions of the simulation cell are very slow and gradual. The perturbation introduced in the motion of the grains, in comparison to the more familiar case of a fixed container, is very small.

\section{Rigidity and stiffness matrices}

We introduce here the appropriate formalism and state the relevant properties of static contact networks. It is implied throughout this section that small displacements about an equilibrium configurations are dealt with to first order (as an infinitesimal motion, i.e. just like velocities), and related to small increments of applied forces, moments and stresses. In the following we shall exploit the definitions of stiffness matrices $\underline{\underline{K}}^{(1)}$ (Eqn. 18) and $\underline{\mathbf{K}}^{(2)}$ to discuss stability properties of packings. The

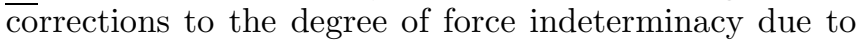
free mechanism motions, as expressed by relations (19) or (20), will also be used.

The properties are stated in a suitable form to the periodic boundary conditions with controlled diagonal stress components, as used in our numerical study.

\section{Definition of stiffness matrix}

We consider a given configuration with bead center positions $\left(\mathbf{r}_{i}, 1 \leq i \leq n\right)$ and orientations $\left(\theta_{i}, 1 \leq i \leq n\right)$, and cell dimensions $\left(L_{\alpha}, 1 \leq \alpha \leq 3\right)$. The grain center displacements $\left(\mathbf{u}_{i}\right)_{1 \leq i \leq n}$ are conveniently written as

$$
\mathbf{u}_{i}=\tilde{\mathbf{u}}_{i}-\underline{\underline{\epsilon}} \cdot \mathbf{r}_{i}
$$

with a set of displacements $\tilde{\mathbf{u}}_{i}$ satisfying periodic boundary conditions in the cell with the current dimensions, and the elements of the diagonal strain matrix $\underline{\epsilon}$ express the relative shrinking deformation along each $\overline{\bar{d}}$ irection, $\epsilon_{\alpha}=-\Delta L_{\alpha} / L_{\alpha}$. Gathering all coordinates of particle (periodic) displacements and rotation increments, along with strain parameters, one defines a displacement vector in a space with dimension equal to the number of degrees of freedom $N_{f}=6 n+3$,

$$
\mathbf{U}=\left(\left(\tilde{\mathbf{u}}_{i}, \Delta \theta_{i}\right)_{1 \leq i \leq n},\left(\epsilon_{\alpha}\right)_{1 \leq \alpha \leq 3}\right) .
$$

Let $N_{c}$ denote the number of intergranular contacts. In every contacting pair $i-j$, we arbitrarily choose a "first" grain $i$ and a "second" one $j$. The normal unit vector $\mathbf{n}_{i j}$ points from $i$ to $j$ (along the line joining centers for spheres). The relative displacement $\delta \mathbf{u}_{i j}$ is defined for spherical grains with radius $R$ as

$$
\delta \mathbf{u}_{i j}=\tilde{\mathbf{u}}_{i}+\delta \theta_{i} \times R \mathbf{n}_{i j}-\tilde{\mathbf{u}}_{j}+\delta \theta_{j} \times R \mathbf{n}_{i j}+\underline{\underline{\epsilon}} \cdot \mathbf{r}_{i j}
$$

in which $\mathbf{r}_{i j}$ is the vector pointing from the center of the first sphere $i$ to the nearest image of the center of the second one $j$. The normal part $\delta \mathbf{u}_{i j}^{N}$ of $\delta \mathbf{u}_{i j}$ is the increment of normal deflection $h_{i j}$ in the contact. (10) defines a $3 N_{c} \times N_{f}$ matrix $\underline{\mathbf{G}}$ which transforms $\mathbf{U}$ into the $3 N_{c}$-dimensional vector of relative displacements at contacts $\delta \mathbf{u}$ :

$$
\delta \mathbf{u}=\underline{\underline{\mathbf{G}}} \cdot \mathbf{U}
$$


In agreement with the literature on rigidity theory of frameworks [46] ( $-\underline{\underline{\mathbf{G}}}$ is termed normalized rigidity matrix in that reference), we call $\underline{\underline{\mathbf{G}}}$ the rigidity matrix.

In each contact a force $\mathbf{F}_{i j}$ is transmitted from $i$ to $j$, which is split into its normal and tangential components as $\mathbf{F}_{i j}=N_{i j} \mathbf{n}_{i j}+\mathbf{T}_{i j}$. The static contact law (without viscous terms) expressed in Eqns. (1), (3), with the conditions stated in Section IIA, relates the $3 N_{c}$-dimensional contact force increment vector $\Delta \mathbf{f}$, formed with the values $\Delta N_{i j}, \Delta \mathbf{T}_{i j}$ of the normal and tangential parts of all contact force increments, to $\delta \mathbf{u}$ :

$$
\Delta \mathbf{f}=\underline{\underline{\mathcal{K}}} \cdot \delta \mathbf{u}
$$

This defines the $\left(3 N_{c} \times 3 N_{c}\right)$ matrix of contact stiffnesses $\underline{\underline{\mathcal{K}}} . \underline{\mathcal{K}}$ is block diagonal (it does not couple different contacts), and is conveniently written on using coordinates with $\mathbf{n}_{i j}$ as the first basis unit vector. In simple cases the $3 \times 3$ block of $\underline{\underline{\mathcal{K}}}$ corresponding to contact $i, j, \underline{\underline{\mathcal{K}}}_{i j}$ is diagonal itself and contains stiffnesses $K_{N}\left(h_{i j}\right)$ and (twice in 3 dimensions) $K_{T}\left(h_{i j}\right)$ as given by (2) and (3):

$$
\underline{\underline{\mathcal{K}}}_{i j}=\left[\begin{array}{ccc}
K_{N}\left(h_{i j}\right) & 0 & 0 \\
0 & K_{T}\left(h_{i j}\right) & 0 \\
0 & 0 & K_{T}\left(h_{i j}\right)
\end{array}\right] .
$$

More complicated non-diagonal forms of $\underline{\underline{\mathcal{K}}}_{i j}$, which actually depend on the direction of the increments of relatives displacements in the contact, are found if friction is fully mobilized (which does not happen in well-equilibrated configurations), or corresponding to those small motions reducing the normal contact force. The effects of such terms is small, with our choice of parameters, and is discussed in paper III [38].

External forces $\mathbf{F}_{i}$ and moments $\boldsymbol{\Gamma}_{i}$ (at the center) applied to the grains, and diagonal Cauchy stress components $\Sigma_{\alpha}$ can be gathered in one $N_{f}$-dimensional load vector $\mathbf{F}^{\mathrm{ext}}$ :

$$
\mathbf{F}^{\mathrm{ext}}=\left(\left(\mathbf{F}_{i}, \boldsymbol{\Gamma}_{i}\right)_{1 \leq i \leq n},\left(\Omega \Sigma_{\alpha}\right)_{1 \leq \alpha \leq 3}\right),
$$

chosen such that the work in a small motion is equal to $\mathbf{F}^{\text {ext }}$. U. The equilibrium equations - the statements that contact forces $\mathbf{f}$ balance load $\mathbf{F}^{\text {ext }}$ - is simply written with the tranposed rigidity matrix, as

$$
\mathbf{F}^{\mathrm{ext}}={ }^{\mathbf{T}} \underline{\underline{\mathbf{G}}} \cdot \mathbf{f}
$$

This is of course easily checked on writing down all force and moment coordinates, as well as the equilibrium form of stresses:

$$
\Omega \Sigma_{\alpha}=\sum_{i<j} F_{i j}^{\alpha} r_{i j}^{\alpha}
$$

As an example, matrices $\underline{\underline{\mathbf{G}}}$ and ${ }^{\mathbf{T}} \underline{\underline{\mathbf{G}}}$ were written down in [47] in the simple case of one mobile disk with 2 contacts with fixed objects in 2 dimensions, the authors referring to $-{ }^{\mathbf{T}} \mathbf{\underline { G }}$ as the "contact matrix". The same definitions and matrices are used in [48] in the more general case of a packing of disks.
Returning to the case of small displacements associated with a load increment $\Delta \mathbf{F}^{\text {ext }}$, one may write, to first order in $\mathbf{U}$,

$$
\Delta \mathbf{F}^{\mathrm{ext}}=\underline{\underline{\mathbf{K}}} \cdot \mathbf{U},
$$

with a total stiffness matrix $\underline{\underline{\mathbf{K}}}$, comprising two parts, $\underline{\underline{\mathbf{K}}}^{(1)}$ and $\underline{\underline{\mathbf{K}}}^{(2)}$, which we shall respectively refer to as the constitutive and geometric stiffness matrices. $\underline{\underline{\mathbf{K}}}^{(1)}$ results from Eqns. 11, 12 and 15.

$$
\underline{\underline{\mathbf{K}}}^{(1)}={ }^{\mathbf{T}} \underline{\underline{\mathbf{G}}} \cdot \underline{\underline{\mathcal{K}}} \cdot \underline{\underline{\mathbf{G}}} .
$$

$\underline{\underline{\mathbf{K}}}^{(2)}$ is due to the change of the geometry of the packing. Its elements (see Appendix B), relatively to to their counterparts in $\underline{\underline{\mathbf{K}}}^{(1)}$, are of order $F / K_{N} R \sim h / R$, and therefore considerably smaller in all practical cases. The constitutive stiffness matrix is also called "dynamical matrix" [31, 41]. One advantage of decomposition (18) is to separate out the effects of the contact constitutive law, contained in $\underline{\mathcal{K}}$ and those of the contact network, contained in $\underline{\underline{\mathbf{G}}} \cdot \overline{\overline{\mathbf{G}}}$ is sensitive in general to the orientations of normal unit vectors $\mathbf{n}_{i j}$ and to the "branch vectors" joining the grain centers to contact positions - which reduce to $R \mathbf{n}_{i j}$ for spheres of radius $R . \underline{\underline{\mathbf{K}}}^{(2)}$, on the other hand, unlike $\underline{\underline{\mathbf{G}}}$, is sensitive to the curvature of grain surfaces at the contact point [49, 50].

\section{Properties of the rigidity matrix}

To the rigidity matrix are associated the concepts (familiar in structural mechanics) of force and velocity (or displacement) indeterminacy, of relative displacement compatibility and of static admissibility of contact forces. Definitions and properties stated in [32] for frictionless grains, straightforwardly generalize to packings with friction.

The degree of displacement indeterminacy (also called degree of hypostaticity [32]) is the dimension $k$ of the

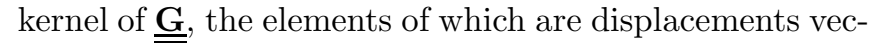
tors $\mathbf{U}$ which do not create relative displacements in the contacts: $\delta \mathbf{u}=0$. Such displacements are termed (firstorder) mechanisms. Depending on boundary conditions, a grain packing might have a small number $k_{0}$ of "trivial" mechanisms, for which the whole system moves as one rigid body. In our case, attributing common values of $\tilde{\mathbf{u}}$ to all grains gives $k_{0}=3$ independent global rigid motions.

The degree of force indeterminacy $\mathrm{H}$ (also called degree of hyperstaticity [32]) is the dimension of the kernel of ${ }^{\mathbf{T}} \underline{\underline{\mathbf{G}}}$, or the number of independent self-balanced contact force vectors. If the coordinates of $\mathbf{f}$ are regarded as the unknowns in system of equations (15), and if $\mathbf{F}^{\text {ext }}$ is supportable, then there exists a whole H-dimensional affine space of solutions.

From elementary theorems in linear algebra one deduces a general relation between $\mathrm{H}$ and $k$ [32]

$$
N_{f}+\mathrm{H}=3 N_{c}+k .
$$


An isostatic packing is defined as one devoid of force and velocity indeterminacy (apart from trivial mechanisms). Excluding trivial mechanisms (thus reducing $N_{f}$ to $N_{f}-k_{0}$ ), and loads that are not orthogonal to them, one then has a square, invertible rigidity matrix. To any load corresponds a unique set of equilibrium contact forces. To any vector of relative contact displacements corresponds a unique displacement vector.

With frictionless objects, in which contacts only carry normal forces, it is appropriate to use $N_{c}$-dimensional contact force and relative displacement vectors, containing only normal components, and to define the rigidity matrix accordingly [32]. Then (19) should be written as

$$
N_{f}+\mathrm{H}=N_{c}+k \text {. }
$$

In the case of frictionless spherical particles, all rotations are mechanisms, hence a contribution of 3 to $k$. Thus one may in addition ignore all rotations, and subtract $3 n$ both from $N_{f}$ and from $k$, so that (20) is still valid. In such a case, the rigidity matrix coincides (up to a sign convention and normalization of its elements) with the one introduced in central-force networks, trusses and tensegrity structures 51]. Donev et al., in a recent publication on sphere packings [52], call rigidity matrix what we defined as its transpose ${ }^{\mathbf{T}} \underline{\underline{\mathbf{G}}}$.

\section{Control parameters}

The geometry and the mechanical properties of sphere packings under given pressure $P$ depends on a small set of control parameters, which can be conveniently defined in dimensionless form [23, 53].

Such parameters include friction coefficient $\mu$ and viscous dissipation parameter $\zeta$, which were introduced in Sec. IIA.

The elastic contact law introduces a dimensionless stiffness parameter $\kappa$, which we define as:

$$
\kappa=\left(\frac{\tilde{E}}{P}\right)^{2 / 3} .
$$

Note that $\kappa$ does not depend on bead diameter $a$. Under pressure $P$, the typical force in a contact is of order $P a^{2}$. It corresponds to a normal deflection $h$ such that $P a^{2} \sim$ $\tilde{E} \sqrt{a} h^{3 / 2}$ due to the Hertz law (1). Therefore, $\kappa$ sets the scale of the typical normal deflection $h$ in Hertzian contacts, as $h / a \sim 1 / \kappa$.

In the case of monodisperse sphere packings in equilibrium in uniform state of stress $\underline{\underline{\sigma}}$, pressure $P=\operatorname{tr} \underline{\underline{\sigma}} / 3$ is directly related to the average normal force $\langle N\rangle$. $\overline{\text { Let }}$ us denote as $\Phi$ the solid fraction and $z$ the coordination number $\left(z=2 N_{c} / n\right)$. As a simple consequence of the classical formula for stresses recalled in Sec. IIB (Eqn. 8 in the static case, or Eqn. (16), one has, neglecting contact deflections before diameter $a$,

$$
P=\frac{z \Phi\langle N\rangle}{\pi a^{2}},
$$

whence an exact relation between $P$ and contact deflections:

$$
\frac{\left\langle h^{3 / 2}\right\rangle}{a^{3 / 2}}=\frac{\pi}{z \Phi \kappa^{3 / 2}}
$$

The limit of rigid grains is approached as $\kappa \rightarrow \infty$. $\kappa$ can reach very high values for samples under their own weight, but most laboratory results correspond to levels of confining pressure in the $100 \mathrm{kPa}$ range. Experimental data on the mechanical properties of granular materials in quasistatic conditions below a few tens of $\mathrm{kPa}$ are very scarce (see, however, [54] and [55]). This is motivated by engineering applications (100 $\mathrm{kPa}$ is the pressure below a few meters of earth), and this also results from difficulties with low confining stresses. Below this pressure range, stress fields are no longer uniform, due to the influence of the sample weight, and measurements are difficult (e.g., elastic waves of measurable amplitude are very strongly damped).

We set the lowest pressure level for our simulation of glass beads to $1 \mathrm{kPa}$ or $10 \mathrm{kPa}$, which corresponds to $\kappa \simeq 181000$ and $\kappa \simeq 39000$. Such values, as we shall check, are high enough for some characteristic properties of rigid sphere packings to be approached with good accuracy. Upon increasing $P$, the entire experimental pressure range will be explored in the two companion papers [37, 38].

Another parameter associated with contact elasticity is the ratio of tangential to normal stiffnesses (constant in our model), related to the Poisson ratio of the material the grains are made of. Although we did not investigate the role of this parameter, several numerical studies 41, 56] showed its influence on global properties to be very small.

The "mass" $M$ of the global degrees of freedom is chosen to ensure slow and gradual changes in cell dimensions, and dynamical effects are consequently assessed on comparing the strain rate $\dot{\epsilon}$ to intrinsic inertial times, such as the time needed for a particle of mass $m$, initially at rest, accelerated by a typical force $P a^{2}$, to move on a distance a. This leads to the definition of a dimensionless inertia parameter :

$$
I=\dot{\epsilon} \sqrt{m / a P}
$$

The quasistatic limit can be defined as $I \rightarrow 0 . \quad I$ was successfully used as a control parameter in dense granular shear flows [57, 58, 59], which might be modelled on writing down the $I$ dependence of internal friction and density [60, 61].

The sensitivity to dynamical parameters $I$ and $\zeta$ should be larger in the assembling stage (as studied in the present paper) than in the subsequent isotropic compression of solid samples studied in paper II [37], for which one attempts to approach the quasi-static limit. In the following we will assess the influence of parameters $\mu, \kappa$, $I$ and $\zeta$ on sample states and properties. 


\section{LOW-PRESSURE ISOTROPIC STATES OF FRICTIONLESS PACKINGS.}

\section{A. Motivations}

Numerical samples are most often produced by compression of an initially loose configuration (a granular gas) in which the grains do not touch. If the friction coefficient is set to zero at this stage, one obtains dense samples, which depend very little on chosen mechanical parameters. These frictionless configurations are in a particular reference state which was recently investigated by several groups [31, 52]. We shall dwell on such an academic model as assemblies of rigid or slightly deformable frictionless spheres in mechanical equilibrium for several reasons. First, we have to introduce various characterizations of the microstructure of sphere packings that will be useful in the presence of friction too. Then, such systems possess rather specific properties, which are worth recalling in order to assess whether some of them could be of relevance in the general case. Frictionless packings also represent, as we shall explain, an interesting limit case. Finally, one of our objectives is to establish the basic uniqueness, in the statistical sense, of the internal state of such packings under isotropic, uniform pressures, provided crystallization is thwarted by a fast enough dissipation of kinetic energy.

\section{B. Assembling procedures}

\section{Previous results}

Since we wish to discuss a uniqueness property, we shall compare our results to published ones whenever they are available. Specifically, we shall repeatedly refer to the works of O'Hern, Silbert, Liu and Nagel [31], and of Donev, Torquato and Stillinger [52], hereafter respectively abbreviated as OSLN and DTS. Both are numerical studies of frictionless sphere packings under isotropic pressures.

OSLN use elastic spheres, with either Hertzian or linear contact elasticity. They control the solid fraction $\Phi$, and record the pressure at equilibrium. Their samples (from a few tens to about 1000 spheres) are requested to minimize elastic energy at constant density. For each one, pressure and elastic energy vanish below a certain threshold packing fraction $\Phi_{0}$, which is identified to the classical random close packing density. Above $\Phi_{0}$, pressure and elastic constants are growing functions of density. OSLN report several power law dependences of geometric and mechanical properties on $\Phi-\Phi_{0}$ which we shall partly review.

DTS differ in their approach, as unlike OSLN (and unlike us) they use strictly rigid spherical balls, and approach the density of equilibrated rigid, frictionless sphere packing from below. They use a variant of the classical (event-driven) hard-sphere molecular dynamics method [44, 62], in which sphere diameters are continuously growing, the Lubachevsky-Stillinger (LS) algorithm [63, 64], to compress the samples. DTS's approximation of the strictly rigid sphere packing as the limit of a hard sphere glass with very narrow interstices (gaps) between colliding neighbors (contact forces in the static packing are then replaced by transfers of momentum between neighbors), and their resorting to linear optimization methods [32, 65], enable them to obtain very accurate results in samples of 1000 and 10000 beads.

DTS expressed doubts as to whether numerical "soft" (elastic) sphere systems could approach the ideal rigid packing properties, and both groups differ in their actual definition of jamming and on the relevance and definition of the random close packing concept. Relying on our own simulation results, we shall briefly discuss those issues in the following.

\section{Frictionless samples obtained by $M D$}

Our numerical results on packings assembled without friction are based on five different configurations of $n=4000$ beads prepared by compression of a granular gas without friction. First, spheres are placed on the sites of an FCC lattice at packing fraction $\Phi=0.45$ (below the freezing density, $\Phi \simeq 0.49[66])$. Then they are set in motion with random velocities, and left to interact in collisions that preserve kinetic energy, just like the molecules of the hard-sphere model fluid studied in liquid state theory [44, 66]. We use the traditional eventdriven method [62], in a cubic cell of fixed size, until the initial crystalline arrangement has melted. Then, velocities are set to zero, and the molecular dynamics method of Section III is implemented with an external pressure equal to $10 \mathrm{kPa}$ for glass beads $(\kappa \simeq 39000)$. Energy is dissipated thanks to viscous forces in contacts, and the packing approaches an equilibrium state. Calculations are stopped when the net elastic force on each particle is below $10^{-4} a^{2} P$, the elastic contributions to the stress components equal the prescribed value $P$ with relative error smaller than $10^{-4}$ and the kinetic energy per particle is below $10^{-8} \mathrm{~Pa}^{3}$. On setting all velocities to zero, it is observed that the sample does not regain kinetic energy beyond that value, while the unbalanced force level does not increase. We have thus a stable equilibrium state. This is further confirmed by the absence of mechanism in the force-carrying contact network, apart from the trivial free translational motion of the whole set of grains as one rigid body. From (18), mechanisms coincide with "floppy modes" of the constitutive stiffness matrix $\underline{\underline{\mathbf{K}}}^{(1)}$ (i.e., the elements of its kernel). The geometric stiffness $\underline{\mathbf{K}}^{(2)}$, as checked in Appendix B, is a very small correction (compared to those of $\underline{\underline{K}}^{(1)}$ the elements of matrix $\underline{\underline{\mathbf{K}}}^{(2)}$ are of order $\kappa^{-1}$ ).

In the following, such configurations assembled without friction will be referred to as A states. 
In order to check for a possible influence of the assembling procedure on the final configurations, we simulated another, similar sample series, denoted as A', for which the LS algorithm was used to bring the solid fraction from 0.45 to 0.61 , before equilibrating at the desired pressure with Hertzian sphere molecular dynamics.

Observed geometric and mechanical characteristics of $\mathrm{A}$ and $\mathrm{A}^{\prime}$ states are reported below and compared to other published results, in particuler those of OSLN and DTS. We also state specific properties of rigid frictionless sphere packings, to which A configurations at high $\kappa$ are close. Unlike OSLN, we use pressure or stiffness level $\kappa$ as the control parameter. The state OSLN refer to as "point J", which appears as a rigidity threshold $\Phi=\Phi_{0}$ if solid fraction $\Phi$ is used as the control parameter, is approached here as $\kappa \rightarrow \infty$.

\section{Compression rates and duration of agitation stage}

Molecular dynamics is not the fastest conceivable route to minimize the sum of elastic and potential energies, and the MD approach does not necessarily find the nearest minimum in configuration space. For that purpose, the direct conjugate gradient minimization approach, as used by OSLN, which involves no inertia and follows a path of strictly decreasing energy in configuration space is the best candidate.

However, the time scales involved in the MD simulations can be compared to experimental ones. In simulations, A configurations approach their final density within a few tens of time $\tau=\sqrt{m /(a P)}$, and come to their final equilibrium with a few hundreds of $\tau$. Comparable laboratory experiments in which dense samples are assembled are sample preparation with the pluviation or rain deposition technique, in which grains are deposited at constant flow rate under gravity, with a constant height of free fall [33, 34, 67, 68]. Such an assembling technique produces homogeneous samples. Grains are first agitated near the free surface, and then subjected to a quasistatic pressure increase as pouring procedes. The relevant pressure scale corresponds therefore to the weight of the agitated superficial layer of the sample being assembled [67], typically of the order of 10 diameters, hence $P \sim 10 \mathrm{mg} / \mathrm{a}^{2}$ and $\tau \sim \sqrt{a /(10 \mathrm{~g})}$, about $3 \times 10^{-3} \mathrm{~s}$ for $a=1 \mathrm{~mm}$. Approximating the compaction time by the time needed to renew entirely the agitated superficial layer, we obtain a few times $10^{-2} \mathrm{~s}$ if this time is to be of order $10 \tau$, as in our simulations. This corresponds to a fraction of a second to fill up a $10 \mathrm{~cm}$ high container, a value within the experimental range. The main conclusion from this crude analysis is that laboratory assembling processes are rather fast, with typical compaction times similar to those of our simulated isotropic compression procedure.

On the other hand, the LS procedure followed by DTS, which we used to produce our A' samples, unavoidably involves many collisions and a considerable level of agi- tation while particle diameters grow at a prescribed rate. In practice, kinetic energy actually increases on implementing the LS algorithm: receding velocities after a collision have to be artificially increased in order to make sure particles that are continuously growing in diameter actually move apart after colliding [63, 64]. Velocities have to be scaled down now and then for computational convenience, a feature the actual compacting process, depending on the ratio of growing rate to quadratic velocity average, is sensitive to. In our implementation there were typically 110 collisions per sphere in the range $0.49 \leq \Phi \leq 0.58$ (the most dangerous interval for crystal nucleation [66, 69]), and 90 collisions for $0.58 \leq \Phi \leq 0.61$. DTS report using expansion rates of $10^{-4}$, while ours started as $10^{-2}$, in units of the quadratic mean velocity.

Consequently, the order of simulation results, from the fastest, least agitated case to the slowest one is as follows: first the OSLN results, then our A, followed by our A' series, and finally the simulations by DTS (who used a slower implementation of the LS method than our A' one).

\section{Energy minimization and density}

\section{What is "jamming"?}

In spite of a long tradition of studies on the geometry of sphere assemblies, the connection between mechanical equilibrium and density maximization has seldom been stressed. This property was presented, in slightly different forms, in the mathematics [70] and physics [32, 52] literature. It is worth recalling it here, as the purpose of this work is to discuss both geometric and mechanical properties of such particle packings. This connection is simply expressed on noting that configurations of rigid, frictionless, non-adhesive spherical particles in stable equilibrium under an isotropic confining pressure are those that realize a local minimum of volume in configuration space, under the constraint of mutual impenetrability. It is no wonder then that the isotropic compaction of frictionless balls is often used as a route to obtain dense samples [27, 30]. In DTS [52] and in other works by the same group [65, 71], the authors use a definition of strictly jammed configurations of hard particles as those for which particles cannot move without interpenetrating or increasing the volume of the whole system. Their definition is therefore exactly equivalent to that of a stable equilibrium state with rigid, frictionless grains under an isotropic confining pressure.

If we now turn to elastic, rather than rigid, spherical particles, with Hertzian contacts as defined in Sec. II. then stable equilibrium states under given pressure $P$ are local minima of the potential energy defined as $(H$ denotes the Heavside step function)

$$
W=P \Omega+\sum_{1 \leq i<j \leq n} \frac{2 \tilde{E} \sqrt{a}}{15} h_{i j}^{5 / 2} H\left(h_{i j}\right) .
$$


As stiffness parameter $\kappa$ increases, the second term of (24) imparts an increasing energetic cost to elastic deflections $h_{i j}$, and the solution becomes an approximation to a minimum of the first term, with impenetrability constraints, i.e., a stable equilibrium state of rigid, frictionless balls. The value of $\kappa$ is an indicator of the distance to the ideal, rigid particle configuration, and it is arguably more convenient to use that the density, used by OSLN, because it does not vary between different samples. OSLN had to adjust the density separately for each sample in order to approach the limit of rigid grains, so that the pressure approached zero, corresponding to a rigidity threshold. Their definition of jamming is based on a local minimum of elastic energy, and therefore also coincides with ours: a jammed state is a stable equilibrium state.

\section{Solid fractions}

Our A configurations have a solid fraction $\Phi=$ $0.6370 \pm 0.0015$ (indicated error bars correspond throughout the paper to one sample-to-sample standard deviation). We shall check below that the small density difference between $\kappa=39000$ and $\kappa \rightarrow \infty$ is much smaller than the statistical uncertainty on $\Phi$. OSLN performed a careful statistical analysis of finite size effects and uncertainty on $\Phi$, leading to estimates shown on Fig. 1] Fig. 1also shows another MD data point we obtained for $n=1372$. Our $\Phi$ values coincide with OSLN's estimation of size-dependent averages and fluctuations, once it is extrapolated to larger sample sizes (or, possibly, our configurations are very slightly denser). Our A' samples exhibit higher densities than A ones, $\Phi=0.6422 \pm 0.0002$ - a fairly small difference, but clearly larger than error bars. DTS do not report $\Phi$ values very precisely, but mention solid fractions in the range 0.625 to 0.63 [52, page 7], on excluding the volume of rattlers, particles that transmit no force. This entails $0.639 \leq \Phi \leq 0.644$ once those inactive grains, which represent about $2.2 \%$ of the total number, are taken into account. LS-made samples were shown in 10] to jam, depending on the compression rate, over the whole solid fraction range between 0.64 and the maximum value $\pi /(3 \sqrt{2})$ corresponding to the perfect FCC crystal. The final values of the solid fraction therefore correlate with the duration of the agitation stage in the assembling procedure. The RCP density is traditionnally associated with a minimization of crystalline order. In the next section we check for indications of incipient crystalline order in A and A' samples.

\section{Traces of crystalline order}

The possible presence of crystal nuclei, the FCC and HCP lattices (the former the more stable thermodynamically) and hybrids thereof being the densest possible arrangements, is a recurring issue in sphere packing studies.

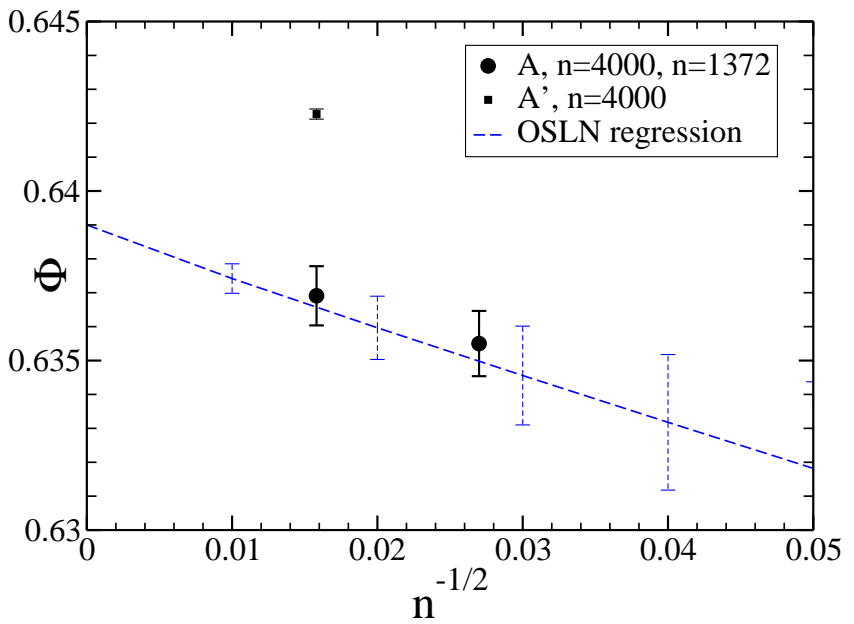

FIG. 1: (Color online) Solid fraction $\Phi$ versus $n^{-1 / 2}$. Blue dotted line: average value, with standard deviation indicated as error bars, according to OSLN's results, extrapolated to $n \rightarrow \infty$. Black round dots with error bars: our A samples for $n=4000$ and other, very similar results for $n=1372$. Square dot: A' samples with $n=4000$ (this point has a smaller error bar).

A recent numerical study of crystallization dynamics in the hard sphere fluid is that of Volkov et al. [66], in which the authors used several indicators and measures of incipient crystalline order that we apply here to A and A' states. First, bonds are defined as (fictitious) junctions between the centers of neighboring spheres if their distance is smaller than some threshold, often chosen as corresponding to the first minimum in pair corelation function $g(r)$ (about $1.4 a$ in our case, see Sec. IIIF 1). Then, a local order parameter is associated to each grain $i$, as:

$$
Q_{l}^{\mathrm{loc}}(i)=\left[\frac{4 \pi}{2 l+1} \sum_{m=-l}^{m=l}\left|\hat{q}_{l m}(i)\right|^{2}\right]^{1 / 2},
$$

in which $\hat{q}_{l m}(i)$ is an average over all neighbors $j$ of $i$ numbered from 1 to $N_{b}(i)$, the number of bonds of $i$ :

$$
\hat{q}_{l m}(i)=\frac{1}{N_{b}(i)} \sum_{j=1}^{N_{b}(i)} Y_{l m}\left(\mathbf{n}_{i j}\right),
$$

$\mathbf{n}_{i j}$ denoting as usual the unit vector pointing from the center of $i$ to the center of $j$.

$Q_{4}$ and $Q_{6}$, in particular, have been used to distinguish different local orders [7, 66, 69]. In the sequel we use the average $Q_{6}^{\text {loc }}$ of (26) over all grains, as well as a global parameter $Q_{6}$, defined on taking the average over all bonds within the sample, instead of those of a particular grain $i$ in (25). The values of those parameters are given in table I Global $Q_{6}$ values are small in large samples, because they tend to average to zero in the presence of randomly oriented polycrystalline textures. They can be used nevertheless to observe crystallization in samples 
of $\sim 10000$ beads, as they finally reach values comparable to the perfect crystal one 69].

Next, following [66], we normalize the set $\left(\hat{q}_{l m}(i)\right)_{-l \leq m \leq l}$, on multiplying, for any given $l$ and $i$, each of its $2 l+1$ components by an appropriate common factor thus obtaining $\left(\bar{q}_{l m}(i)\right)_{-l \leq m \leq l}$, such that

$$
\sum_{m=-l}^{m=l}\left|\bar{q}_{l m}(i)\right|^{2}=1 .
$$

If the values $\hat{q}_{l m}(i)$ are viewed as the components of a $2 l+$ 1-dimensional local order parameter, then $\bar{q}_{l m}(i)$ might be viewed as its "phase" or "angular" part, characteristic of the choice of a direction, rather than of the intensity or extent with which the system is locally ordered. Then a bond is termed crystalline if it joins two particles for which those "phases" are sufficiently correlated: (the star indicates complex conjugation)

$$
\left|\sum_{m=-l}^{m=l} \bar{q}_{l m}(i) \bar{q}_{l m}^{*}(j)\right| \geq 0.5 .
$$

A particle is said to be in a crystalline configuration if at least 7 of its bonds (out of 12.5-13, see table【) is "crystalline", according to definition (28) with $l=6$. One may check how numerous those particles are and whether they tend to cluster in crystalline regions. Table [ contains those various indicators, as observed in samples of type $\mathrm{A}$ and A' at the largest studied stiffness level. Order parameters have a very small value, indicating as expected a large distance to crystal order. Only a small fraction of bonds and grains are declared "crystalline" according to the above definitions. However, it does transpire from the data of table I that A' states are consistently more "ordered" than A ones, with a small, but systematic difference for all listed indicators (see also Appendix D). Most notable is the increase of the size of "crystalline" regions. A direct visualization of those domains, as we checked, shows that they are quite far from perfectly ordered, but reveals some local tendency to organization in parallel stacked layers, and to the formation of $2 \mathrm{D}$ triangular lattice patterns within the layers. Luchnikov et al. 69] report simulation of 16000 particle samples of the hard sphere fluid evolving towards crystallization at constant density (between $\Phi=0.55$ and $\Phi=0.6$ ), as monitored by the global $Q_{6}$ parameter and the distribution of local $Q_{6}$ values. They observed, like Volkov et al. 66], that several thousands of collisions per particle were necessary for a significant evolution to take place, which is compatible with our observation of a detectable, but very small tendency with about 100 collisions per particle with our A' samples.

$Q_{6}^{\text {loc }}$ and $Q_{4}^{\text {loc }}$, as defined in (25), were also used by Aste et al. [7] to characterize local arrangements, in an experimental study of sphere packing geometry by X-ray tomography. These results rely on observations of large samples of tens to hundreds of thousands of beads, although not isotropic. Particles are classified according to the pair of values $Q_{6}^{\mathrm{loc}}(i), Q_{4}^{\mathrm{loc}}(i)$. We compared the geometry of our numerical samples of similar density to those experimental data, with the result that although the most frequently observed values of $Q_{6}^{\mathrm{loc}}(i)$ and $Q_{4}^{\mathrm{loc}}(i)$ were quite close to experimental ones in dense samples, and the proportion of hcp-like particles were similar, fcclike local environments were exceptional in simulations, whereas a few percent of the spheres were classified in that category in the experimental results. Quantitative results are given in Appendix D. Nucleation of crystalline order is strongly sensitive to sample history and boundary conditions [66].

\section{E. Properties of force networks}

\section{Identification and treatment of rattlers}

The rattlers are defined as the grains that do not participate in carrying forces and remain, therefore, free to "rattle" within the cage formed by their forcecarrying, rigidly fixed neighbors. We refer to the network of contacting grains that carry forces as the backbone. The backbone is the structure formed by nonrattler grains. The fraction $x_{0}$ of rattlers at $\kappa=39000$ is $x_{0}=0.013 \pm 0.002$ in A samples, and it is slightly higher, $x_{0}=0.018 \pm 0.002$ in $\mathrm{A}^{\prime}$ ones. DTS report $x_{0} \simeq 0.022$, and hence once again our A' results are closer to theirs. The proportion of rattlers increases slightly for stiffer contacts (higher $\kappa$ values).

Distinguishing between the backbone and the rattlers requires some care, as very small forces on the backbone might be confused with forces below tolerance between rattler and backbone grains, or between two rattlers. We apply the following simple procedure. First, we regard as rattlers all spheres having less than four contacts: less than three contacts implies a mechanism, and only three is impossible if forces are all strictly compressive. We also discard from the backbone all spheres with only forces smaller than the tolerance. Then, all the contacts of eliminated spheres being also removed, other spheres might (although this is an extremely rare occurrence) have less than four contacts, so the procedure is iterated (twice at most is enough in our samples, although one such sweep is usually enough) until no more rattler is detected. We found this method to work correctly for $n=4000$ and $\kappa=39000$. If one eliminates too many particles, the identified backbone might become floppy (hypostatic). We check, however, that its constitutive stiffness matrix remains positive definite, thereby avoiding such pitfall. The proportion of rattlers is likely to increase for stiffer contacts (higher $\kappa$ ).

The presence of rattlers complicates the analysis of geometric properties of static packings, because their positions are not determined by the equilibrium requirement. The rattlers are free to move within a "cage" formed by their backbone neighbors, and there is no obvious way in principle to prefer one or another of their infinitely many 


\begin{tabular}{|cc|ccccccc|}
\hline State $d_{c} / a$ & $Z$ & $Z_{c r}$ & $Q_{6}$ & $Q_{6}^{\text {Ioc }}$ & $Q_{6}^{\text {Ioc,cr }}$ & $x_{c r}$ & $\left\langle n_{c r}\right\rangle$ \\
\hline \hline $\mathrm{A}$ & 1.35 & $12.36 \pm 0.03$ & $2.91 \pm 0.06$ & $(1.7 \pm 0.3) \times 10^{-2}$ & $0.392 \pm 0.001$ & $0.417 \pm 0.003$ & $0.080 \pm 0.005$ & 19.8 \\
\hline $\mathrm{A}^{\prime}$ & 1.35 & $12.50 \pm 0.02$ & $3.13 \pm 0.11$ & $(1.9 \pm 0.5) \times 10^{-2}$ & $0.398 \pm 0.0005$ & $0.420 \pm 0.002$ & $0.104 \pm 0.006$ & 54.8 \\
\hline $\mathrm{A}$ & 1.40 & $13.11 \pm 0.02$ & $2.94 \pm 0.06$ & $(1.6 \pm 0.3) \times 10^{-2}$ & $0.370 \pm 0.001$ & $0.394 \pm 0.002$ & $0.083 \pm 0.006$ & 22.8 \\
\hline $\mathrm{A}^{\prime}$ & 1.40 & $13.20 \pm 0.02$ & $3.16 \pm 0.11$ & $(1.8 \pm 0.3) \times 10^{-2}$ & $0.377 \pm 0.0006$ & $0.397 \pm 0.003$ & $0.103 \pm 0.006$ & 64.5 \\
\hline
\end{tabular}

TABLE I: Indicators of possible incipient crystalline order in states A and A' at $\kappa=39000$. $Z$ is the coordination number of first heighbors, $Z_{c r}$ the "crystalline bond" coordination number, $Q_{6}$ and $Q_{6}^{\text {loc }}$ the global and (average) local order parameters, $Q_{6}^{\text {loc,cr }}$ its average value within crystalline regions, $x_{c r}$ the fraction of "crystalline" particles and $\left\langle n_{c r}\right\rangle$ the mass average of the number of particles in a "crystalline cluster". First neighbors are defined here as those closer than distance $d_{c}=1.35 a$ or $d_{c}=1.40 a$, near the first minimum in $g(r)$.

possible positions. This renders the evaluation of geometric data like pair correlations somewhat ambiguous. Moreover, rattlers, although scarce in frictionless packings, can be considerably more numerous in frictional ones (see Section IV]). We therefore specify whether the results correspond to direct measurements on the configurations resulting from the simulations, with rattlers floating in some positions resulting from compaction dynamics, or whether rattlers have been fixed, each one having three contacts with the backbone (or some previously fixed other rattler). To compute such fixed rattler positions with $\mathrm{MD}$, we regard each backbone grain as a fixed object, exert small isotropically distributed random forces on all rattlers and let them move to a final equilibrium position (assuming frictionless contacts). A third possibility is to eliminate rattlers altogether before recording geometric data. These are three choices referred to as I, II and III in the sequel, and we denote observed quantities with superscripts I, II or III accordingly.

Packings under gravity, if locally in an isotropic state of stress, are expected to be in the same internal state and to exhibit the same properties as the ones that are simulated here. In such a situation, individual grain weights are locally, within an approximately homogeneous subsystem, dominated by the externally imposed isotropic pressure. There is no rattler under gravity, but some grains are simply feeling their own weight, or perhaps that of one or a few other grains relying on them. Such grains are those that would be rattlers in the absence of gravity. Instead of freely floating within the cage of their backbone neighbors, they are supported by the cage floor. The situation should therefore be similar to that of our samples after all rattlers have been put in contact with the force-carrying structure (treatment II), except that the small external forces applied to them are all directed downwards.

\section{Coordination numbers}

Table II gives the distribution of local coordination number values among the spheres for A and A' states at $\kappa=39000$. In this table, $x_{i}$ is the proportion of grains with $i$ contacts. If rattlers are stuck to the back-

\begin{tabular}{|c|cccccccccccc|}
\hline State & $x_{0}$ & $x_{1}$ & $x_{2}$ & $x_{3}$ & $x_{4}$ & $x_{5}$ & $x_{6}$ & $x_{7}$ & $x_{8}$ & $x_{9}$ & $x_{10}$ & $x_{11}$ \\
\hline \hline A (I) & 1.3 & 0 & 0 & 0 & 11.1 & 23.2 & 28.4 & 22.6 & 10.3 & 2.8 & 0.3 & 0.02 \\
\hline A' $^{\prime}(\mathrm{I})$ & 1.8 & 0 & 0 & 0 & 11.6 & 22.5 & 28.2 & 22.3 & 10.6 & 2.8 & 0.3 & 0.01 \\
\hline A (II) & 0 & 0 & 0 & 1.1 & 10.7 & 22.7 & 28.2 & 22.9 & 10.9 & 3.1 & 0.3 & 0.02 \\
\hline A' $^{\prime}(\mathrm{II})$ & 0 & 0 & 0 & 1.7 & 10.9 & 21.9 & 28.2 & 22.5 & 11.4 & 3.1 & 0.3 & 0.01 \\
\hline
\end{tabular}

TABLE II: Percentages $x_{i}$ of grains having $i$ contacts in A and A' configurations, on ignoring the rare contacts with or between rattlers (I), or on fixing the rattlers onto the backbone with small (randomly oriented) forces (II).

bone (method II), one records slightly changed proportions of spheres with $n \geq 3$ contacts, to which values observed within samples under gravity should be compared. Distributions of local coordination numbers observed by DTS coincide to the data of Table II within $1 \%$. We attribute this small difference to the influence of contact deflections of order $\kappa^{-1} a$ in the MD results, while the DTS results are closer to ideally rigid packings (approached as open gaps tend to zero).

\section{Isostaticity}

We now discuss how the isostaticity property 32, 72, 73, 74, 75, 76, 77, 78] of equilibrium states of rigid, frictionless spheres, influences high $\kappa$ configurations of type A.

Isostaticity is a property of the backbone, i.e. the force-carrying contact network, in equilibrium packings of rigid, frictionless spheres. It means that such networks are both devoid of hyperstaticity (force indeterminacy) and of hypostaticity (displacement indeterminacy), apart from possible trivial motions in which all force-carrying grains move as one rigid body. These two properties have different origins [32], and are not valid under the same assumptions. The absence of hyperstaticity $(\mathrm{H}=0$ with the notations of Sec. IICl) results from the generic disorder of the packing geometry. It would hold true for arbitrarily shaped rigid particles interacting by purely normal contact forces whatever the sign of those forces, and it applies to the whole packing, whatever the contacts the rattlers might accidentally have with the backbone. The absence of hypostaticity property (except for trivial 
mechanisms, $k=k_{0}$ ), on the other hand, is only guaranteed for spherical particles with compressive forces in the contacts, and it applies to the sole backbone.

Due to the isostaticity property, the coordination number should be equal to 6 in the rigid limit on the backbone. If $N_{c}$ is the number of force-carrying contacts, then the global (mechanical) coordination number is $z=\frac{2 N_{c}}{n}$ (possible contacts of the rattlers are discarded), and the backbone coordination number is defined as $z^{*}=\frac{2 N_{c}}{n\left(1-x_{0}\right)}=\frac{z}{1-x_{0}} . \quad z^{*}$, rather than $z$, has the limit 6 as $\kappa \rightarrow+\infty$. In A samples $(\kappa=39000)$ one has $z^{*}=6.074 \pm 0.002$ (and hence $z \simeq 5.995$ ), the excess over the limit $z^{*}=6$ resulting from contacts that should open on further decreasing the pressure.

The isostaticity property can be used to evaluate the density increase due to finite particle stiffness. To first order in the small displacements between $P=0$ (or $\kappa=+\infty$ ) and the current finite pressure state $\mathrm{A}$, one might use the theorem of virtual work 32], with the displacements that bring all overlaps $h_{i j}$ to zero, and the current contact forces. Such motions leading to a simultaneous opening $\left(h_{i j}=0\right)$ of all contacts are only possible on networks with no hyperstaticity, because there is no compatibility condition on relative normal displacements 32]. This yields an estimate of the increase of the solid fraction $\Delta \Phi$ over its value $\Phi_{0}$ in the rigid limit, as

$$
\frac{1}{\Omega} \sum_{i j} N_{i j} h_{i j}=P \frac{\Delta \Phi}{\Phi} .
$$

This equality can be rearranged using the Hertz contact law (11) to relate $N_{i j}$ to $h_{i j}$, and relation (22). We denote as $Z(\alpha)$ the moment of order $\alpha$ of the distribution of normal forces $N_{i j}$, normalized by the average over all contacts:

$$
Z(\alpha)=\frac{\left\langle N^{\alpha}\right\rangle}{\langle N\rangle^{\alpha}}
$$

(29) can be rewritten as:

$$
\Delta \Phi=3^{5 / 3} \Phi^{1 / 3} Z(5 / 3)\left(\frac{\pi}{z}\right)^{2 / 3} \kappa^{-1} .
$$

In the isostatic limit which is approached at large $\kappa$, the force distribution and its moments are determined by the network geometry, and we observed $Z(5 / 3)=1.284$. Taking for $z$ and $\Phi$ the values at the highest studied stiffness level $\kappa(\kappa=39000)$, this enables us to evaluate the density change between those configurations and the rigid limit as $\Delta \Phi \simeq 1.15 \times 10^{-4}$. As announced before this is smaller than the statistical uncertainty on $\Phi$, and hence this does not improve our estimation of the solid fraction $\Phi_{0}$ of the packing of rigid particles $(\kappa=+\infty)$. Recalling $\kappa^{-1}=(P / \tilde{E})^{2 / 3}$, (31) means that the macroscopic relation between pressure and density has the same power law form $\left(P \propto(\Delta \Phi)^{3 / 2}\right)$ as the contact law $\left(N \propto h^{3 / 2}\right)$. This was observed by OSLN. It would hold, because of the isostaticity property in the rigid limit, for whatever exponent $m$ in the contact law, the prefactor of the macroscopic relation $P \propto(\Delta \Phi)^{m}$ involving $Z(1+1 / m)$, a moment of the geometrically determined force distribution.

As a consequence of (31), one can simply relate the bulk modulus of frictionless packings to the pressure, as observed by OSLN too, a property which will be used and discussed in paper III [38], which deals with elasticity of packings.

\section{Force distribution}

The force distribution we observe in A samples at high $\kappa$ values approaches the one of a rigid packing, which due to isostaticity is a purely geometric property. It is represented on Figure 2. The data presented here are av-

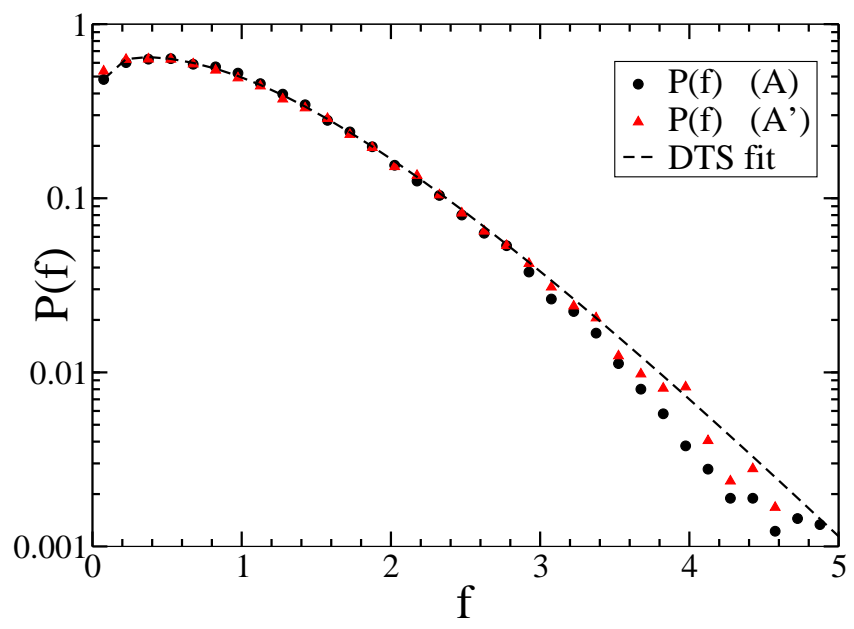

FIG. 2: (Color online) Probability distribution function $P(f)$ of normalized contact forces $f=N /\langle N\rangle$ in A and A' configurations at high $\kappa$. The dashed line is the fit proposed by DTS: $P(f)=\left(3.43 f^{2}+1.45-1.18 /(1+4.71 f)\right) \exp (-2.25 f)$.

eraged over 5 samples. Because of relation (22), all samples prepared at the same pressure have the same average force, and this restores the "self-averaging" property, which OSLN observed to be lacking on using solid fraction instead of pressure as the control parameter. The choice of $\Phi$ as a state variable, because of the finite size of the sample causing fluctuations of the threshold $\Phi_{0}$ where $P$ vanishes, is less convenient in that respect.

Fig. 2 also shows that the form proposed by DTS to fit their data is in very good agreement with our results, except perhaps for large forces, for which it is a better fit for A' data - thus providing additional evidence that A' samples are closer than A ones to the DTS results. 


\section{F. Geometric characterization}

\section{Pair correlation function}

Pair correlations should preferably be measured either with method I or method II, as there is no reason to eliminate rattlers before studying geometric properties. Comparisons between pair correlation functions $g^{I}(r)$ and $g^{I I}(r)$ (Fig. 3) show very little difference on the scale of one particle diameter. Results of Fig. 3] are very similar to other published ones (e.g., in DTS), with an apparent divergence as $r \rightarrow a$ and a split second peak, with sharp maxima at $r=a \sqrt{3}$ and $r=2 a$. The pair correlation

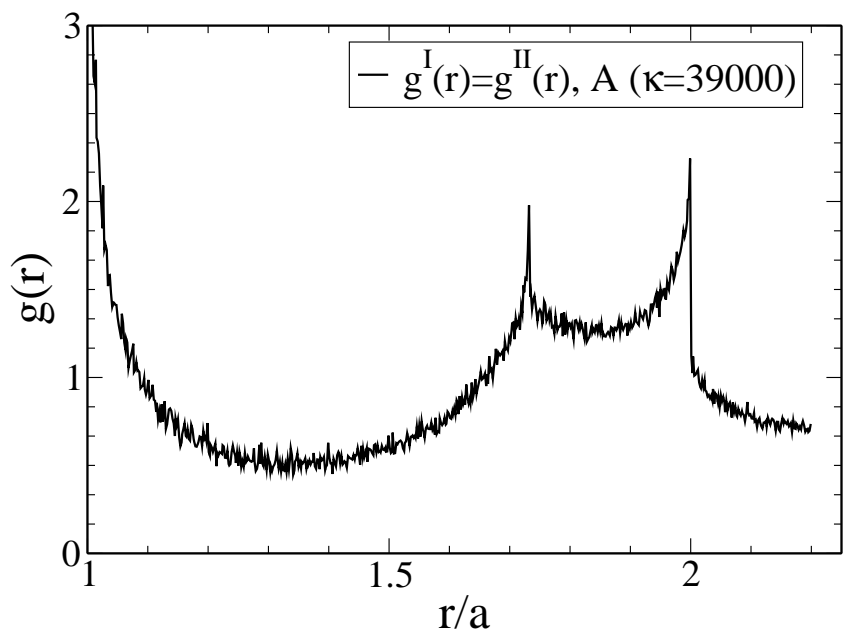

FIG. 3: Pair correlation functions $g^{I}(r)$ and $g^{I I}(r)$ versus $r / a$ in A samples at $\kappa=39000$. Both definitions coincide on this scale (only the peak for $r \rightarrow a^{+}$is slightly different).

function should contain a Dirac mass at $r=a$ in the limit of rigid grains, which broadens into a sharp peak for finite contact stiffness. The weight of this Dirac term or peak in the neighbor intercenter distance probability distribution function $4 \pi \frac{n}{\Omega} r^{2} g(r)=24\left(r^{2} / a^{3}\right) \Phi g(r)$ is coordination number $z$, and the shape of the left shoulder of the peak at finite $\kappa$ is directly related to the force distribution $P(N)$ :

$$
(\text { For } \delta>0) g(a-\delta)=\frac{z a^{3} \tilde{E} \sqrt{a \delta}}{48 \Phi(a-\delta)^{2}} P\left(\frac{\tilde{E} \sqrt{a} \delta^{3 / 2}}{3}\right) .
$$

This explains the observation by OSLN [31] of the width of the $g(r)$ peak decreasing approximatively as $\Delta \Phi$, while its height increases as $(\Delta \Phi)^{-1}$, as the threshold density $\Phi_{0}$ is approached from above. The form of the distribution of contact forces, which is determined by the geometry of the isostatic backbone, remains exactly the same for all small enough values of $\Delta \Phi$, with a scale factor proportional to $\Delta \Phi^{3 / 2}$, due to Eqns. 22 and 31 .

The sharp drop of $g(r)$ at $r=a \sqrt{3}$ and $r=2 a$ was found by DTS to go to a discontinuity in the rigid particle limit. This can be understood as follows. Each sphere has a number of first contact neighbors ( $z$ on average) at distance $r=a$ if the grains are rigid, and a number of second contact neighbors (i.e. particles not in contact with it, but having a contact with at least one of its first contact neighbors). Such second contact neighbors will make up for a significant fraction of particles with their centers at a distance $r \leq 2 a$, but none of them can be farther away. Futhermore, this leads to a systematic depletion of the corona $2 a<r<2 a+\delta$ (with $0<\delta<a$ ) by steric exclusion.

\section{Near neighbor correlations}

As $r \rightarrow a^{+}$, pair correlations are conveniently expressed with the gap-dependent coordination number $z(h) . \quad z(h)$ is the average number of neighbors of one sphere separated by an interstice narrower than $h . z(0)$ is the usual contact coordination number $z$. Function $z(h)$ has three possible different definitions $z^{I}, z^{I I}$ and $z^{I I I}$ according to the treatment of rattlers. All three of them were observed to grow as $z(0)+C h^{0.6}$ for $h$ smaller than about $0.3 a$, constant $C$ taking slightly different values for $z^{I}, z^{I I}$ and $z^{I I I}$. $z^{I I I}(h)$ is equal to $z^{*}$ for $h=0$, and is very well fitted with the value $C=11$ found by DTS $[52$, Fig. 8]. $z(h)$ deviates from this power law dependence corresponding to the rigid limit for small $h$, of the order of the typical overlap $\kappa^{-1}$, as shown on Fig. 4. This power law corresponds to $g(r)$ diverging as $(r-a)^{-0.4}$ as $r \rightarrow a^{+}$. Silbert et al., in a recent numerical study

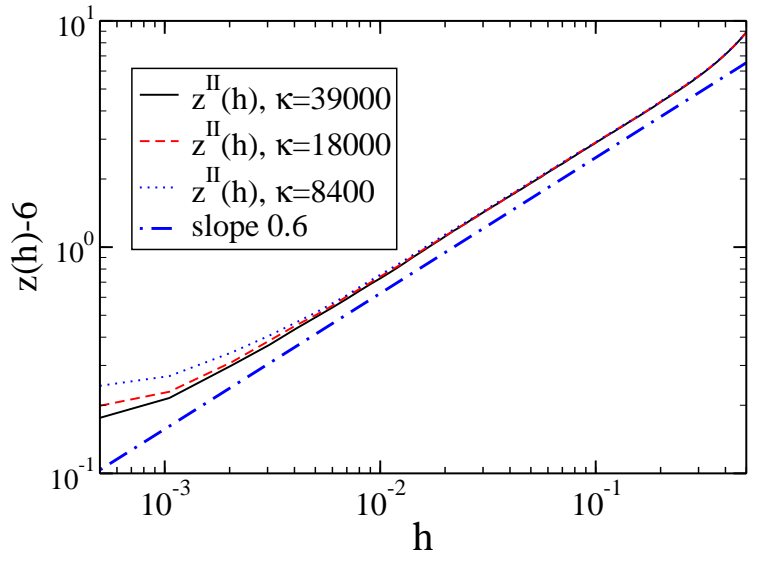

FIG. 4: (Color online) Coordination number of near neighbors function of interstice $h$. The power law regime extends to smaller and smaller $h$ values as $\kappa$ increases.

of states with high levels of rigidity $[79]\left(\kappa>10^{6}\right)$, report observing $z^{I}(h)$ to grow with an exponent closer to 0.5 , although somewhat dependent on the choice of the $h$ interval for the fit. However this does not contradict our main conclusion that different numerical approaches track the same RCP state in the rigid limit. 


\section{Other properties of contact networks}

Ignoring rattlers (method III) one may record the density of specific particle arrangements in the backbone. We thus find the contact network (joining all centers of interacting particles by an edge) to comprise a number of equilateral triangles, such that on average each backbone grain belongs to $2.04 \pm 0.04$ triangles. In the rigid limit this gives a Dirac term for $\theta=\pi / 3$ in the distribution of angles $\theta$ between pairs of contacts of the same grain. Tetrahedra are however very scarce (as observed by DTS), involving about $2.5 \%$ of the beads, and pairs of tetrahedra with a common triangle are exceptional (5 such pairs in 5 samples of 4000 beads). Pairs of triangles sharing a common base are present with a finite density, which explains the discontinuous drop at $r=a \sqrt{3}$ of $g(r)$, this being the largest possible distance for such a population of neighbor pairs.

\section{G. Conclusions}

We summarize here the essential results of Section [II] about frictionless packings.

\section{Uniqueness of the RCP state}

Our numerical evidence makes a strong case in favor of the uniqueness of the simulated rigid packing state made with frictionless spheres under isotropic pressure. Specifically, we observed quantitative agreements with other published results [31, 52 in the coordination numbers, the force distributions, the pair correlations and the frequency of occurrence of local contact patterns, even though different numerical methods have been used. The small remaining differences in solid fraction, proportion of rattlers, and probability of large contact forces all correlate with the duration of agitated assembling stage, which can be measured in terms of numbers of collisions per grain at a given density. This duration directly correlates to the packing fraction and to the small amount of crystalline order in the samples. We therefore checked in an accurate, quantitative way the traditional views about random close packing (RCP). The RCP state can be defined in practice as the unique state in which rigid frictionless spherical beads assemble in a static equilibrium state under isotropic pressure, in the limit of fast compression, so that the slow evolution towards crystallization has a negligible influence. The LubachevskyStillinger algorithm tends to produce packings with a small but notable crystalline fraction.

\section{Relevance of MD simulations, role of micromechanical parameters}

The uniqueness of the RCP implies that dynamical parameters $\zeta$ and $I$ have no influence on the frictionless packing structures, at least in the limit of fast compression rates.

Standard MD methods compare well with specifically designed methods that deal with rigid particles, and prove able to approach the rigid limit with satisfactory, if admittedly smaller, accuracy. Recalling that $\kappa=39000$ corresponds to glass beads under $10 \mathrm{kPa}$, it seems that laboratory samples under usual conditions might in principle (if friction mobilization can be suppressed) approach the ideal (rigid particle) RCP state.

Moreover, the time scales to assemble samples in MD simulations, if compared to estimated preparation times in the laboratory with such techniques as controlled pluviation, has the right order of magnitude. This means that the assembling proces is rather fast in experimental practice when grains are deposited under gravity, which explains why densities above RCP are not directly obtained. Of course, in practice, many procedures produce anisotropic states. Anisotropic packings of rigid, frictionless balls, under other confining stresses than a hydrostatic pressure, should differ from the RCP state, and the numerical simulations of gravity deposited packings of frictionless beads of refs. [24, 25] could be analysed in this respect. We chose here to study ideal preparation methods, and we only deal with isotropic systems.

\section{Approach to isostaticity in the rigid limit}

We checked that bead packings under typical laboratory pressures such as $10 \mathrm{kPa}$ might closely approach the isostaticity property of rigid frictionless packings. We showed that some observations made by O'Hern et al. 31. on pressure or bulk modulus dependence on density, and on the shape of the first peak of $g(r)$ were direct consequences of this remarkable property.

\section{LOW-PRESSURE STATES OF FRICTIONAL PACKINGS OBTAINED BY DIFFERENT PROCEDURES}

\section{A. Introduction}

It is well known that the introduction of friction in granular packings tends in general to reduce density and coordination number, as observed in many recent numerical simulations (see e.g. 24, 25, 26, 27]), and that frictional granular assemblies, unlike frictionless ones, can be prepared in quite a large variety of different states. In the field of soil mechanics, sand samples are traditionnally classified by their density [11, 12, 13], which determines behaviors that have been observed in simulations 
of model systems as well [21, 27]. (Inherent anisotropy of the fabric, i.,e., the one due to the assembling process, rather than induced by anisotropic stresses, is a secondary, less influential state variable [14, 15]). Engineering studies on sands usually resort to a conventional definition of minimum and maximum densities, based on standardized procedures 80].

The motivation of the present study is to explore the range of accessible packing states, as obtained by different numerical procedures that produce homogeneous and isotropic periodic samples. We therefore chose to bypass the painstaking computations needed to mimic actual laboratory assembling methods, but we argue that our procedures produce plausible structures with similar properties.

One key result is that density alone does not determine the internal state of an isotropic packing, because the coordination number can vary independently.

The A-type configurations obtained without friction in Section IVB are local density maxima in configuration space (see Sec. III C 1). Hence compaction methods can be regarded as strategies to circumvent the mobilization of intergranular friction forces. Two such procedures are studied here, in a simplified, idealized form: lubrication and vibration. We also simulate, as a reference, a state which can be regarded as a loose packing limit, at least with a definition relative to one assembling method and friction coefficient $\mu=0.3$; and we prepare, as an interesting limit from a theoretical point of view, infinite friction samples.

Assembling procedures are described in Section IVB geometric aspects are studied in Section IVC and contact network properties in Section IVD. Section IVE summarizes the results.

\section{B. Assembling processes for frictional grains}

Just like in the frictionless case, for each one of the packing states, we prepare 5 samples of 4000 beads, over which results are averaged, error bars corresponding to sample-to-sample fluctuations. The equilibrium criteria are those of Section ПIB 2, supplemented with a similar condition on moments. To identify rattlers, we use the procedure defined in Section IIIE1 which is adapted to the case of frictional grains: spheres with as few as two contacts may carry forces (even large ones, as we shall see) and should not be regarded as rattlers.

\section{Looser packings compressed with final friction coefficient}

We used direct compression of a granular gas in the presence of friction $(\mu=0.3)$, another standard numerical procedure [26, 27, 28], in which the obtained density and coordination number are decreasing functions of $\mu[24,25,26,27]$. This produces rather loose samples hereafter referred to as $\mathrm{D}$ ( $\mathrm{B}$ and $\mathrm{C}$ ones, to be defined further, denoting denser ones, closer to $\mathrm{A}$, but arguably more "realistic"). D samples were made with exactly the same method as A ones (see Sec. IIIB2), except that the friction coefficient $\mu=0.3$ was used instead of $\mu=0$. In principle, D configurations should depend on initial compaction dynamics: increasing the rate of compression could produce denser equilibrated packings, just like a larger height of free fall, whence a larger initial kinetic energy, increases the density of configurations obtained by rain deposition under gravity 67 . We request the reduced compression rate $I$, defined in (23), not to exceed a prescribed maximum value $I_{\max }$. The choice of $I_{\max }=10^{-3}$ and $\zeta=0.98$ yields solid fractions $\Phi=0.5923 \pm 0.0006$ and backbone coordination numbers $z^{*}=4.546 \pm 0.009$, with a rattler fraction $x_{0}=(11.1 \pm 0.4) \%$. These data correspond to $P=1 \mathrm{kPa}$ (or $\kappa \simeq 181000$ ). Very similar results are obtained on using a different, but low enough pressure, such as 10 or even $100 \mathrm{kPa}$, as remarked in [41] (where 2D samples were assembled by oedometric compression), and as indicated in table [II. However, a quasistatic compression from $P=1 \mathrm{kPa}$ to 10 or $100 \mathrm{kPa}$ produces slightly different states at the same pressure. The influence of $\zeta$ should disappear in the limit of slow compression, $I \rightarrow 0$. A practical definition of a ( $\mu$-dependent) limit of loose packing obtained by direct compression, can therefore be proposed as the $I \rightarrow 0$ limit of our D states. As reported in table III, a value of the damping parameter ten times as small as the standard one $\zeta=0.98$ results in quite similar configuration properties, on compressing a loose granular gas under $P=10 \mathrm{kPa}$, with $I_{\max }=10^{-3}$. So did in fact faster compressions, with $I_{\max }=10^{-1}$, keeping $\zeta=0.98$. The data of Table III thus suggest that we very nearly achieved the independence on dynamical parameters that is expected in the $I \rightarrow 0$ limit with our choice of control parameters. We note, however, that other possible definitions of a random loose packing, such as the one by Onoda and Liniger [81] result in different (smaller) solid fractions. Looser arrangements of equal-sized spherical particles can also be stabilized with adhesive contact forces, e.g. on introducing the capillary attractions produced by the menisci formed by a wetting fluid in the interstices between neighboring grains [82].

In addition to packing fraction $\Phi$, coordination number $z^{*}$, fraction of rattlers $x_{0}$, Table III lists the reduced second moment $Z(2)$ of the normal force distribution, as defined in (30), the proportion of two-coordinated beads (to be discussed in Section IVD), $x_{2}$, and the average values of ratios $\|\mathbf{T}\| / N$ (friction mobilization) among contacts carrying normal forces larger and smaller than the average, respectively denoted as $M_{1}$ and $M_{2}$. As a result of some amount of quasistatic compression of the initial assembly, the width of the force distribution decreases, as witnessed by smaller values of $Z(2)$ in table III and so does the mobilization of friction, as measured by $M_{1}$ and $M_{2}$. The effects of compression on the structure and the forces are further studied in paper II [37]. On comparing numerically simulated loose packings to experi- 
TABLE III: Isotropic states of type D, from direct compression of the granular gas at the indicated pressure (rows marked "gas"), or from gradual, quasistatic compression (rows marked "QS") of solid samples made at the lowest pressure $1 \mathrm{kPa}$ (or $\kappa \simeq 181000$ ). Tests of the influence of viscous dissipation parameter $\zeta$ and maximum value $I_{\text {max }}$ of reduced strain rate in compression are also made for configurations compressed from a granular gas to $10 \mathrm{kPa}$.

\begin{tabular}{|c|c|c|c|c|c|c|c|c|c|c|}
\hline Origin & $\mathrm{P}(\mathrm{kPa})$ & $\zeta$ & $I_{\max }$ & $\Phi$ & $z^{*}$ & $x_{0}(\%)$ & $x_{2}(\%)$ & $Z(2)$ & $M_{1}$ & $M_{2}$ \\
\hline gas & 1 & 0.98 & $10^{-3}$ & $0.5930 \pm 0.0007$ & $4.546 \pm 0.009$ & $11.1 \pm 0.4$ & 2.39 & 1.58 & 0.160 & 0.217 \\
\hline gas & 10 & 0.98 & $10^{-3}$ & $0.5946 \pm 0.0006$ & $4.59 \pm 0.02$ & $10.2 \pm 0.2$ & 2.07 & 1.59 & 0.159 & 0.213 \\
\hline gas & 10 & 0.098 & $10^{-3}$ & $0.5938 \pm 0.0008$ & $4.61 \pm 0.02$ & $10.9 \pm 0.2$ & 1.79 & 1.57 & 0.150 & 0.194 \\
\hline gas & 10 & 0.98 & $10^{-1}$ & $0.5931 \pm 0.0002$ & $4.60 \pm 0.01$ & $10.2 \pm 0.7$ & 1.80 & 1.59 & 0.159 & 0.212 \\
\hline QS & 10 & 0.98 & $10^{-3}$ & $0.5931 \pm 0.0006$ & $4.641 \pm 0.011$ & $10.1 \pm 0.4$ & 2.33 & 1.46 & 0.146 & 0.188 \\
\hline gas & 100 & 0.98 & $10^{-3}$ & $0.5975 \pm 0.001$ & $4.69 \pm 0.02$ & $8.9 \pm 0.5$ & 1.66 & 1.61 & 0.153 & 0.197 \\
\hline QS & 100 & 0.98 & $10^{-3}$ & $0.5936 \pm 0.0006$ & $4.79 \pm 0.02$ & $8.6 \pm 0.4$ & 2.05 & 1.40 & 0.138 & 0.178 \\
\hline
\end{tabular}

ments, it should be recalled that samples are assembled under low pressures in the laboratory: the hydrostatic pressure under a $1 \mathrm{~cm}$ thick layer of glass beads is about $0.15 \mathrm{kPa}$. Numerical configurations under higher confining pressures corresponding to mechanical tests in the laboratory (e.g., sound propagation) are more appropriate models if the testing pressure is significantly larger than the initial, assembling pressure - as for the "QS" samples of table III.

The effects of such proportions of rattlers in granular packings as reported in table III have to our knowledge never been studied in detail. It should be emphasized that this relatively large population of rattlers does not jeopardize the global stability of equilibrium configurations, as the stiffness matrix of the force-carrying network is found devoid of floppy modes (apart from harmless, localized ones associated with two-coordinated particles, to be discussed in Section IVD).

Our D samples should be compared with the simulations reported by Zhang and Makse 83], in which loose sphere packings were also prepared by isotropic compression. Those authors observed, in some cases, lower packing fractions than $\mathrm{D}$ values, $\Phi \simeq 0.57$. Their assembling method is however different: they use a strain-controlled procedure, with a constant compression rate, and then relax the final state at constant volume. In this approach, the pressure reaches very high levels, several orders of magnitude as large as the final value, before samples finally stabilize [83, Fig. 3]. Zhang and Makse report some dependence of the final state on the compressing rate. Once translated into the dimensionless parameter $I$ we have been using here, strain rates used in [83], defined with the typical pressure value $P=100 \mathrm{kPa}$, range between $I=0.1$ and $I=100$. The slowest compression reported in [83] is therefore 100 times as fast as the upper limit for $\dot{\epsilon}$ we have been enforcing in this work. Viscous forces also differ between the present simulations and those of Ref. [83], in which "global damping" terms are used (i.e., forces opposing the individual motion of particles, rather than relative motions).

\section{Use of low friction coefficients: imperfect lubrication}

One way to limit the effects of friction consist in lubricating the grains, as in the experimental study reported in 84]. If all intergranular friction could be suppressed in the assembling procedure, i.e., for perfect lubrication, then the structure of isotropic packings would be the one denoted as A, studied in Section III. The effect of a small friction coefficient in the contacts while the grain assembly is being compressed can be regarded as a crude, simplified model for imperfect lubrication. We made samples, denoted as B, by compressing the granular gas, just like in the $\mathrm{A}$ and $\mathrm{D}$ cases, with $\mu=0.02$. In order to approach the limit of slow compression rates better, we started from D configurations, decreased the friction coefficient to $\mu=0.02$, and then requested that $I<10^{-4}$ while the samples got further compressed to equilibrium under $1 \mathrm{kPa}(\kappa \simeq 181000)$. (In view of the results in the $\mathrm{D}$ case of Section IVB 1, we do not expect the final B state to be sensitive to damping parameter $\zeta$.) We observed that this small friction coefficient had a notable effect on the final solid fraction, as the value $\Phi=0.6270 \pm 2.10^{-4}$ is significantly below the frictionless (A) result, while the coordination number on the active structure is slightly reduced, down to $z^{*}=5.75 \pm 8.10^{-3}$, and the fraction of rattlers raised slightly, to $x_{0}=(1.95 \pm 0.02) \%$.

\section{Dense, frictional packings obtained by shaking}

Another practical strategy to obtain dense configurations is to shake, vibrate or apply repeated "taps" on granular samples [5, 35, 36]. Such procedures involve the introduction of kinetic energy into already quite dense assemblies. In order to investigate their possible effects at a limited computational cost, we avoid the direct simulation of repeated shakes and adopted the following procedure. Starting from the dense A configurations (made without friction and described in Section (III), we first apply a homogeneous expansion, multiplying all coordinates by a common factor $\lambda$ slightly larger than 1 . With equilibrated A states under confinement level $\kappa=39000$, 
the chosen value $\lambda=1.005$ is more than enough to separate all pairs in contact. Then, in order to mimic, in an idealized way, the motion set up by a shaking excitation, the beads are given random velocities (chosen according to a Maxwell distribution), and interact in collisions which preserve kinetic energy, while the volume of the cell is kept constant. This "mixing" stage is simulated with the "hard sphere molecular dynamics" (eventdriven) scheme (just like our initial granular fluids are prepared at $\Phi=0.45$, as described in Section IIIB2). It is pursued until each particle has had $n_{\text {coll }}=50$ collisions on average. The final preparation stage is a fast compression: velocities are set to zero, particles regain their elastic and dissipative properties (as defined in Section IIA, with friction coefficient $\mu=0.3$, and viscous dissipation, $\zeta=0.98$ ), the external pressure $P=10 \mathrm{kPa}$ is applied via the deformable periodic cell, until a final equilibrium is reached.

The final state is hereafter referred to as C. Quite unsurprisingly, its solid fraction, $\Phi=0.635 \pm 0.002$, stays very close to the RCP value obtained in the A state. However, the coordination number is considerably lower, $z^{*}=4.56 \pm 0.03$, which is as small as the value obtained in the loose $(\Phi \simeq 0.593) \mathrm{D}$ state, while the proportion of rattlers raises to $x_{0}=(13.3 \pm 0.5) \%$. Remarkably, on comparing states $\mathrm{B}$ and $\mathrm{C}$, the latter has a higher density, but a much lower coordination number, and a much higher fraction of rattlers.

We did not thoroughly investigate the influence of parameters $\lambda$ and $n_{\text {coll }}$, introduced in the preparation procedure, on the resulting $\mathrm{C}$ states. In the following we focus on configurations obtained with the values $\lambda=1.005$ and $n_{\text {coll }}=50$. Yet, we noted that an increase of $\lambda$ to 1.01 entailed only very slight changes of $\Phi$ and $z^{*}$ (which respectively decreased to 0.633 and 4.54 ), and that the suppression of the "mixing" stage (i.e., setting $n_{\text {coll }}$ to zero) resulted in much higher $z^{*}$ values (around 5.5). Likewise, we did not check for a possible effect of $\zeta$ on the final state. Smaller values than the large one $\zeta=0.98$ used in our simulations of the final compression stage of $\mathrm{C}$ sample preparation are likely to have analogous effects to an increase of the duration of the agitated mixing stage, and should not increase the final coordination number.

\section{Global state variables: summary and discussion}

Table IV gathers some of the parameters characterizing the four different packing states studied in the present paper. ( $M_{1}$ and $M_{2}$ were defined in connection with table III). From table IV configurations with low coordination numbers $(\mathrm{C}$ and $\mathrm{D})$ appear to exhibit a somewhat wider normal force distribution (as measured by $Z(2)$ ), and a significant mobilization of friction, with typical values of $\|\mathbf{T}\| / N$ around $\mu / 2=0.15$ for larger than average normal force components $N\left(M_{1}\right)$, and significantly above $\mu / 2$ for smaller $N$ values $\left(M_{2}\right)$.

The existence of $\mathrm{C}$ states shows that there is no system- atic relationship between density and coordination number, contrarily to some statements in the literature [7]. Of course, for one particular assembling method both quantities will often vary in the same direction as functions of some control parameter. For instance, on preparing samples by deposition under gravity, both density and coordination number are increasing functions of the height of free fall [67]. However, our results show that different preparation methods might lead to contrasting results.

Our results about density and coordination number can be likened to observations made before in numerical models of sphere packings obtained with geometric construction rules [85]. The simplest versions of such algorithms [86, 87], which mimic deposition under gravity, add particles one by one by dropping and rolling them in contact with one or two previously deposited particles, until they are fixed when they rely on three contacts. Those produce packings with $z=6$. More refined versions thereof [85, 88] also involve other, more collective types of moves. The final configurations then contain "bridges" or "arches" [89], defined as sets of particles the final stabilization of which is mutual and collective. In such arches, each grain relies on three others, but some pairs mutually rely, in part, on each other. Those "bridged structures" have much lower coordination numbers, down to about 4.5.

It is not clear, though, to what extent our configurations, which were obtained within a full mechanical model, compare to those that result from such approaches. As shown, e.g., in [90], deposition algorithms based on geometrical rules are supposed to ensure local stability properties, but the resulting granular pilings might turn out to be globally unstable. Moreover, a description of our packings as a sequence of arches placed one after another, assuming it is conceivable, would seem to contradict their homogeneity and isotropy: it is rather arbitrary, in isotropic packings, to regard some particles as "relying" on some others. Such a description was therefore not attempted.

\section{Geometric characterization}

\section{Pair correlation functions}

As observed in previous experimental [7] and numerical 24] results, pair correlation functions present the same features at lower densities as at the largest one $\Phi \simeq 0.64$, in a weakened form, as shown on Fig. 5 . On comparing those functions for states A to D, we observed what follows.

- C samples, obtained from A ones after small rearrangements, exhibit pair correlations that only differ in the detailed shape of the peaks (e.g. below $1.05 a)$, and is indistinguishable elsewhere.

- In spite of the large number of rattlers in samples $\mathrm{C}$ and D, $g^{I}(r)$ and $g^{I I}(r)$ (as defined in Section 
TABLE IV: Isotropic states $(\kappa \simeq 39000$ for $\mathrm{A}$ and $\mathrm{C}, \kappa \simeq 181000$ for $\mathrm{B}$ and $\mathrm{D})$ for different assembling procedures.

\begin{tabular}{|l|ccccccc|}
\hline Procedure & $\Phi$ & $z^{*}$ & $x_{0}(\%)$ & $x_{2}(\%)$ & $\mathrm{Z}(2)$ & $M_{1}$ & $M_{2}$ \\
\hline \hline $\mathrm{A}$ & $0.6370 \pm 0.0002$ & $6.074 \pm 0.0015$ & $1.3 \pm 0.2$ & 0 & 1.53 & 0 & 0 \\
\hline $\mathrm{B}\left(\mu_{0}=0.02\right)$ & $0.6271 \pm 0.0002$ & $5.80 \pm 0.007$ & $1.95 \pm 0.02$ & $\sim 10^{-4}$ & 1.52 & 0.016 & 0.018 \\
\hline $\mathrm{C}(\lambda=1.005)$ & $0.635 \pm 0.002$ & $4.56 \pm 0.03$ & $13.3 \pm 0.5$ & 2.64 & 1.65 & 0.135 & 0.181 \\
\hline $\mathrm{D}$ & $0.5923 \pm 0.0006$ & $4.546 \pm 0.009$ & $11.1 \pm 0.4$ & 2.39 & 1.58 & 0.160 & 0.217 \\
\hline
\end{tabular}

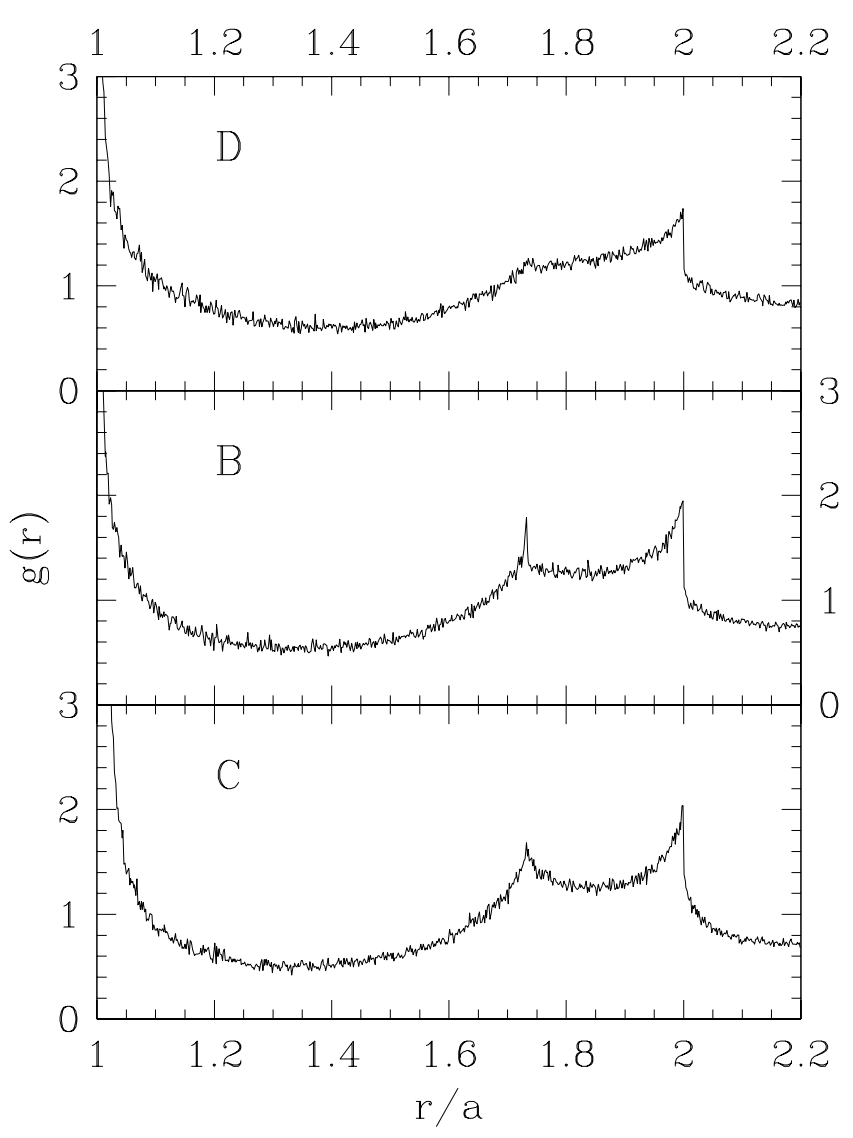

FIG. 5: Pair correlation functions $g(r)$ (definitions $g^{I}(r)$ and $g^{I I}(r)$ coincide on this scale) for configurations B, C, D.

cannot be distinguished on the scale of Fig. 5 .

- The depth of the trough between $r / a=1.1$ and $r / a=1.5$ increases with density.

- The integral below the peaks correlates with density, but the height and sharpness of the drop at $r / a=\sqrt{3}$ and $r / a=2$ correlate with coordination number (which is larger for $\mathrm{B}$ than for $\mathrm{C}$ ), in agreement with the interpretation of such features suggested in Sec. IIIF.

\section{Near neighbor coordination numbers}

The gap-dependent coordination number $z^{I I}(h)$ is shown on Fig. [ for samples A to D. Fig. [6 shows that,

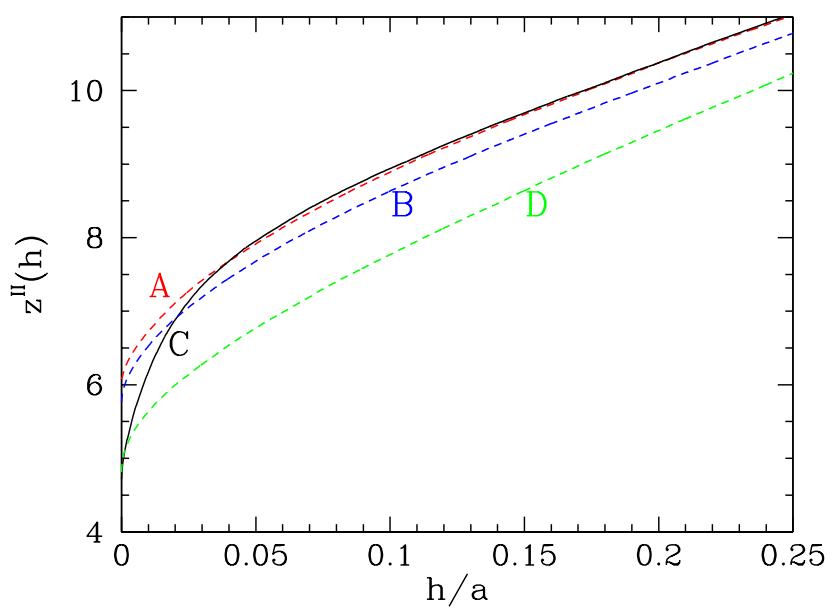

FIG. 6: (Color online) Coordination number for neighbors at distance $\leq h, z^{I I}(h)$, for configurations A (red, upper dashed line), B (blue, middle dashed line), C (black, solid line) and $\mathrm{D}$ (green, bottom dashed line).

as might have been intuitively expected, $z(h)$ correlates with coordination number for small $h$ and with density for larger distances, $h \geq 0.04 a$. We preferably use definition $z^{I I}$, which is obtained on bringing the rattlers in contact with the backbone with small, random forces, as explained in Sec. IIIE $z^{I I}(h)$ can be thought of as more physically meaningful than $z^{I}(h)$, which directly results from the simulation of the packing, and is somewhat ambiguously defined because the positions of the rattlers are not specified. Functions $z^{I I}(h)$ corresponding to $\mathrm{B}$ and C states cross each other for $h \simeq 0.02 a$.

$z^{I}(h)$ and $z^{I I}(h)$ might be fitted by power laws:

$$
\begin{aligned}
z^{I}(h) & =B^{I}+A_{I} h^{\beta_{I}} \\
z^{I I}(h) & =B^{I I}+A_{I I} h^{\beta_{I I}}
\end{aligned}
$$

Figs 7, 8 and 9 display $z^{I}(h)-B^{I}$ and $z^{I I}(h)-B^{I I}$ as functions of $h$ on logarithmic plots for samples D, B and $\mathrm{C}$ (due to the influence, at short distance, of contact deflections on $z(h)$ data, fit parameters $B^{I}$ and $B^{I I}$ are a little smaller than $z$ and $\left.z^{I I}(0)\right)$. For $h$ values smaller than $10^{-4}$, the lowest limit on the axis on Figs. 7,9 and 8 , 
the gap $h$ is of the same order as elastic deflections, $\kappa^{-1}$, and we do not observe simple power laws (as on Fig. 4).

These figures (on which the corresponding values of

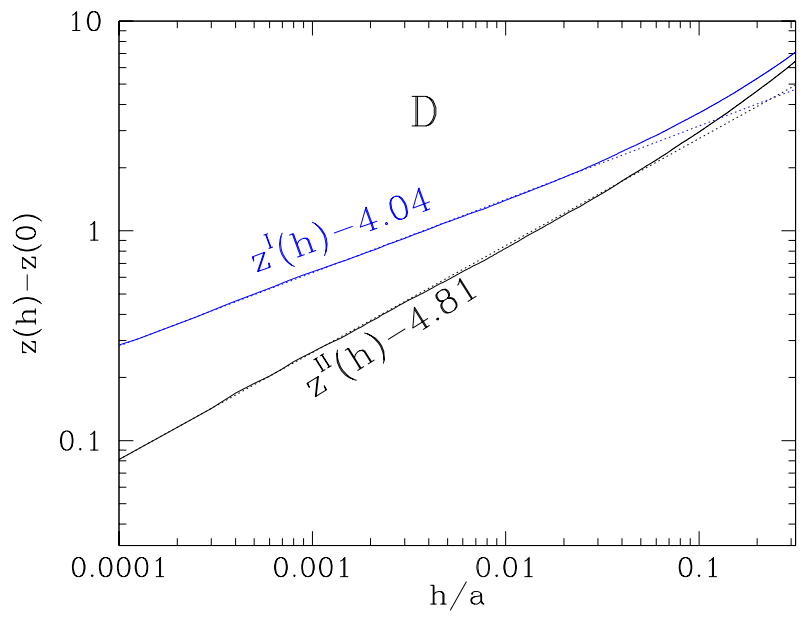

FIG. 7: (Color online) $z^{I}(h)-z$ (black) and $z^{I I}(h)-z^{I I}(0)$ (blue) versus $h$ on doubly logarithmic plot for $\mathrm{D}$ samples at lowest pressure. The slopes of the corresponding dotted straight lines (power law fits) are $\beta_{I I}=0.51$ and $\beta_{I}=0.35$ (see Eqn. 32).

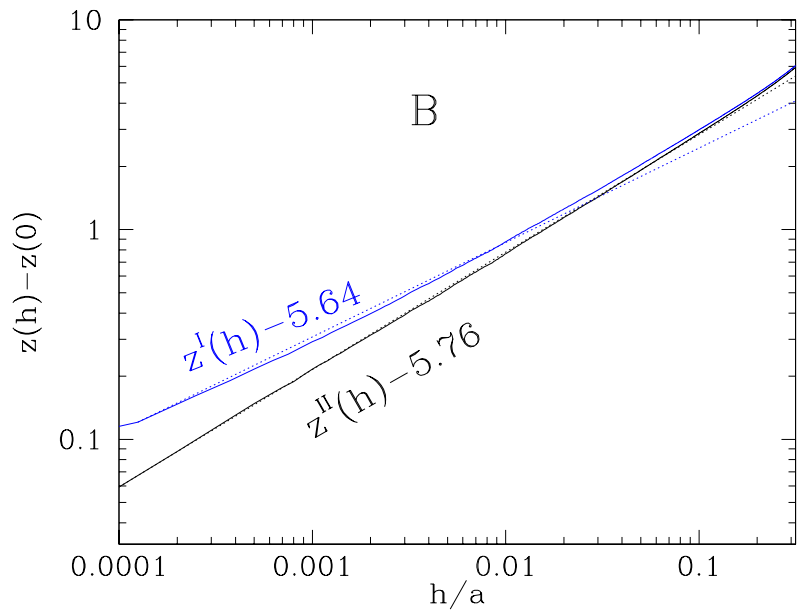

FIG. 8: (Color online) Same as Fig. 7 for B samples. Dotted lines have slopes $\beta_{I I}=0.56$ and $\beta_{I}=0.45$.

$z \simeq z^{I}(0)$ and $z^{I I}(0)$ are also provided) show that $z^{I}$ and $z^{I I}$ have quite different $h$ dependences. This should be accounted for on studying the closing of contacts due to compression (see paper II [37]). As a result of the computation leading to the equilibrated state, many pairs of neighbors end up separated by a very small interstice, so that $z^{I}-z$ already reaches values larger than 0.3 for $h=10^{-4} a$ in samples $\mathrm{C}$ and D. $z^{I}$ then grows with $h$ more slowly than $z^{I I}$, with $\beta_{I I}>\beta_{I}$, and a power law fit of lesser quality. Exponent $\beta_{I I}$, which should be regarded as a more intrinsic quantity than $\beta_{I}$, appears to correlate with solid fraction. It has the same value 0.6 in samples

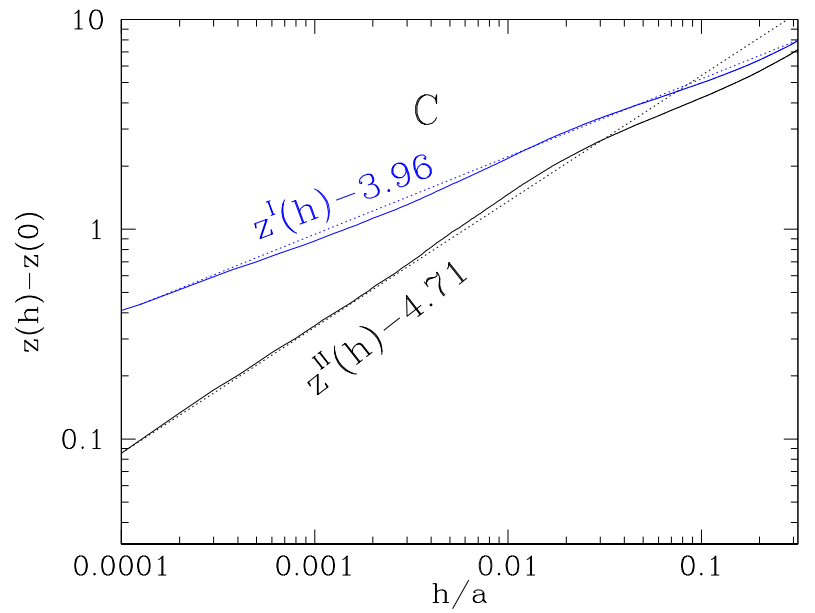

FIG. 9: (Color online) Same as Fig. 7 for C samples. Slopes of dotted straight lines: $\beta_{I I}=0.6$ and $\beta_{I}=0.37$.

$\mathrm{C}$ and A (Figs 9 and 4), decreases to 0.55 in the intermediate density state $\mathrm{B}$, and to 0.51 for the least dense one, D. The power-law form of $z^{I I}$ extends to $h \simeq 0.3$ in configuration $\mathrm{A}$, to about $h \simeq 0.2$ in configuration $\mathrm{B}$, and only to 0.04 and 0.05 for C and D. Such limited power law ranges preclude comparisons with the experimental data of [7].

One has $z^{I}(0) \simeq z$ as a very good approximation, since contacts carrying no force in the equilibrated state obtained by MD are very scarce. $z^{I I}(0)$, on the other hand, is the geometric coordination number once all rattlers are pushed against the backbone. It is larger than the mechanical coordination number, $z$. Specifically, because all rattlers, in treatment II, are dealt with as frictionless, one has:

$$
z^{I I}(0)=z+6 x_{0}-\frac{2 N_{r r}}{n},
$$

in which $N_{r r}$ is the final number of contacts between rattlers once they are positioned against the backbone. Value of $z^{I I}(0)$ can be read on Fig. 6. As pointed out in Section IIIE1, they can be compared to coordination number values of samples under gravity. The results of Silbert et al. [24, Figs. 2 and 3], with $\mu=0.3$, correspond approximately with our D samples: $\Phi \simeq 0.59$ and $z^{I I}(0) \simeq 5$. The systems simulated in [24] are however not isotropic. None of the samples made under gravity in [24, 25] appears to resemble our C state.

\section{Absence of crystalline order, local order parameters}

All indicators of incipient crystalline order given in Table $\prod$ for frictionless A samples take lower values in states $\mathrm{B}$ and $\mathrm{D}$, while $\mathrm{C}$ configurations, due to their geometric proximity, are close to A ones in this respect. Already scant in dense configurations assembled without friction, traces of crystallization are thus negligible in looser ones 
obtained with frictional beads. Like for A samples, numerical data on configurations around one sphere $i$, as characterized by the pair $\left(\hat{Q}_{4}(i), \hat{Q}_{6}(i)\right)$, used by Aste $e t$ al. 7], are presented for states B, C, and D and compared to their experimental results in Appendix D. It is observed that local disordered environments around one grain are very similar in numerical and experimental configurations of equal densities, while local HCP-like arrangements occur with similar (low) frequencies, and FCC-like ones are present in the laboratory, but not detected in simulations.

\section{Properties of force networks}

\section{Local contact coordination numbers}

The distribution of local coordinations is given in Table V, for both mechanical (I) and geometric (II) defini-

\begin{tabular}{|c|ccccccccccc|}
\hline State & $x_{0}$ & $x_{1}$ & $x_{2}$ & $x_{3}$ & $x_{4}$ & $x_{5}$ & $x_{6}$ & $x_{7}$ & $x_{8}$ & $x_{9}$ & $x_{10}$ \\
\hline \hline B (I) & 1.95 & 0 & 0.05 & 0.5 & 16.3 & 26.9 & 27.5 & 18.4 & 7.1 & 1.4 & 0.1 \\
\hline C (I) & 13.3 & 0 & 2.6 & 15.1 & 26.5 & 23.4 & 13.2 & 4.8 & 0.9 & 0.15 & 0 \\
\hline D (I) & 11.1 & 0 & 2.4 & 13.8 & 29.1 & 25.6 & 13.3 & 4.0 & 0.7 & 0.03 & 0 \\
\hline B (II) & 0 & 0 & 0 & 2.1 & 15.3 & 26.5 & 27.5 & 18.9 & 7.8 & 1.6 & 0.2 \\
\hline C (II) & 0 & 0 & 0 & 18.9 & 22.8 & 27.0 & 18.6 & 9.3 & 2.8 & 0.5 & 0.1 \\
\hline D (II) & 0 & 0 & 0 & 17.2 & 25.4 & 29.0 & 18.5 & 7.7 & 1.9 & 0.3 & 0 \\
\hline
\end{tabular}

TABLE V: Percentage $x_{i}$ of grains having $i$ contacts in configurations $\mathrm{B}, \mathrm{C}$ and $\mathrm{D}$, on ignoring contacts with or between rattlers (I), or on fixing them onto the backbone with small, random forces (II).

tions of contacts, for states B to D. Compared to the A case (Table $\amalg$, the distribution is shifted to lower values, with 4 and 5 the most frequent ones (rather than 6 ) in low coordinated packings $\mathrm{C}$ and $\mathrm{D}$. Those samples also have quite a large population of three-coordinated spheres, and a notable one of divalent (two-coordinated) particles. This contrasts with the frictionless case for which $x_{2}=x_{3}=0$. Without friction, divalent spheres, from Eqn. 20, written with $N_{c}=2, N_{f}=3, h=0$, would imply a mechanism and therefore an instability, and threecoordinated ones, in the absence of external forces and cohesion, cannot be equilibrated by non-vanishing normal forces the net effect of which necessarily pushes them away from the plane defined by the three centers of their touching neighbors (the non-generic case with the four sphere centers within the same plane leading to an instability). Spheres with three contacts therefore need some mobilization of friction to transmit non-vanishing forces in an equilibrium configuration, for tangential components are requested to cancel this net repulsion. The Coulomb condition then restricts such possible configurations to flat enough tetrahedra for contact forces to remain within the friction cone. This explains the small value of $x_{3}$ in low friction $(\mu=0.02)$ B samples.

\section{The special case of divalent grains}

With friction, the small structure formed by one sphere having two contacts with fixed objects (Fig. 10), due to Eqn. 19, in which the number of degres of freedom (6) is equal to the number of contact force coordinates, has a degree of force indeterminacy equal to its number of independent mechanisms: $\mathrm{H}=k$. In fact, both numbers are equal to 1 . Self-balanced contact forces (see first part of Fig. 10) are oriented along the line joining the two contacts, just like in the corresponding $2 \mathrm{D}$ case dealt with in [47], and their amplitude is a free parameter (the degree of "wedging" of the grain in the corner formed by its two neighbors [47]). Such a possibility requires in practice that the angle, which we denote as $\alpha$ (Fig. 10), between the line joining the centers of 1 and 2 and the one joining the contact points be smaller than the angle of friction, for the total contact force to stay within the Coulomb cone. In $\mathrm{C}$ and $\mathrm{D}$ samples, we observed $\tan \alpha$ to be distributed rather evenly between 0 and $\mu$, while the intensity of forces transmitted by divalent spheres ranged from 0 to a few times $P \mathrm{P}^{2}$. The mechanism associated
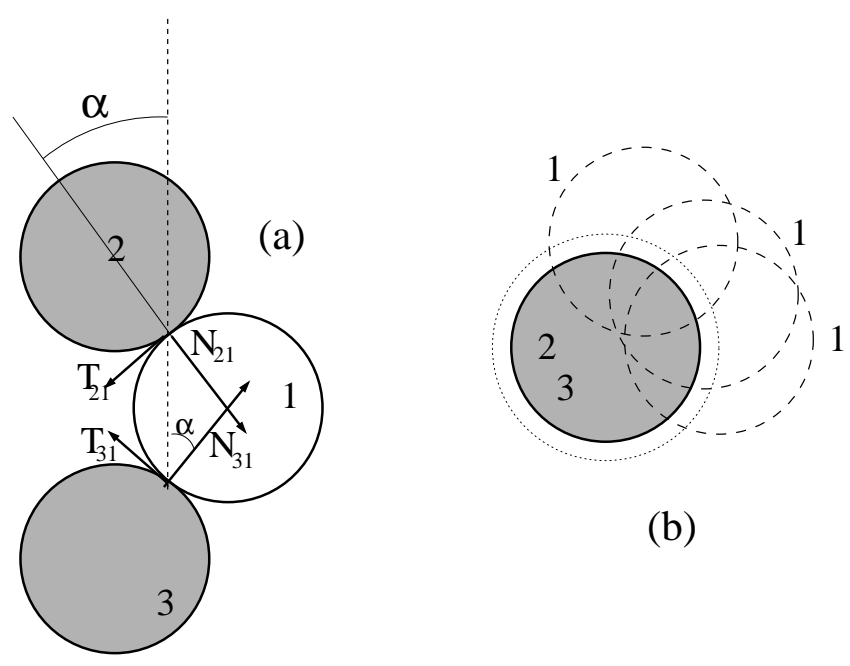

(b)

FIG. 10: Equilibrium and free motion (mechanism) of one sphere (marked 1) with two contacts (with particles marked 2 and 3). (a): in the plane of the three centers, normal and tangential components of the two contact forces balance along the line joining the contact points (dotted line). (b): seen from above, along the direction of the line of the centers of spheres 2 and 3, sphere 1 can move and occupy the different positions depicted with dashed lines, its center describing the dotted circle around the 2-3 axis.

with divalent spheres is a free rolling motion on the two contacts, the line joining the contact points being the instantaneous axis of rotation, as shown on fig. 10(b). In this motion, it is readily checked (see Appendix E) that the rules given in Appendix B for the evolution of contact forces in the case of rolling and pivoting specify that contact forces will remain carried by the line joining the two contacts, with a constant intensity, as such contacts move on the surface of the fixed spheres. Such 
forces will do no work and the kinetic energy of the mobile sphere as well as the elastic energy stored in its two contacts will be kept constant. The equilibrium of the divalent particle is thus marginally stable, with matrix $\underline{\mathbf{K}}^{(2)}$ causing zero acceleration to the free motion. However, such a motion does affect the balance of moments on spheres marked 2 and 3 on Fig. 10, since the constant force is applied at a point that is moving on their surface. Therefore the stability of such free motions, as regards the global contact network, requires some additional analysis - which is tackled in Appendix E. where it is concluded that the packing remains stable. Unlike in frictionless packings [32, 91], mechanisms in the presence of friction do not necessarily lead to instabilities. On building the constitutive stiffness matrix $\underline{\mathbf{K}}^{(1)}$ in the samples we studied, we could check that no other mechanism was present on the backbone than those rolling motions of divalent spheres. Once some stiffness element is introduced to impede the free motion of divalent spheres, one can e.g. check that the Cholesky factorization of the stiffness matrix only involves strictly positive terms on the diagonal.

There can be no contact between two divalent spheres, as the simultaneous equilibrium of each of them with two forces carried by the line joining its two contact points, as on Fig. 10, is impossible.

\section{Degree of force indeterminacy}

On the backbone, with $n\left(1-x_{0}\right)$ spheres and $N_{f}^{*}=$ $6 n\left(1-x_{0}\right)+3$ degrees of freedom, one has on average $z^{*} n\left(1-x_{0}\right) / 2$ contacts and $k=3+x_{2} n$ independent mechanisms, hence a degree of force indeterminacy (hyperstaticity), from (19), given by:

$$
\begin{aligned}
\mathrm{H} & =N_{f}^{*} \frac{z^{*}-z_{0}^{*}}{4}=N_{f}^{*} \frac{z^{* *}-4}{4}, \text { with } \\
z_{0}^{*} & =4-\frac{2 x_{2}}{3\left(1-x_{0}\right)} \text { and } \\
z^{* *} & =z^{*}+\frac{2 x_{2}}{3\left(1-x_{0}\right)}
\end{aligned}
$$

The backbone is devoid of force indeterminacy $(\mathrm{H}=0)$ when its coordination number is equal to $z_{0}^{*}$. Because of the mechanisms associated with divalent beads $z_{0}^{*}$ is strictly smaller than 4. Alternatively, one can define a "corrected" backbone coordination number $z^{* *}$, as written in (34), which is equal to 4 in the absence of force indeterminacy. As to the global mechanical coordination number $z_{0}$ corresponding to the absence of force indeterminacy, its value, given by

$$
z_{0}=4\left(1-x_{0}\right)-\frac{2 x_{2}}{3},
$$

is well below 4. From the data of table $\mathrm{V}, z_{0}$ is about 3.45 in state $\mathrm{C}$ and 3.54 for $\mathrm{D}$ (while $z$ is close to 4 ). Although $\mathrm{H}$ is relatively small compared to the number of degrees of freedom $N_{f}$, the samples with friction and low coordination are still notably hyperstatic - a conclusion shared by other studies [24], which we reach here in the slightly different context of packings in a uniform state of stress. Unlike for frictionless sphere assemblies, there is actually no special reason to expect packings with intergranular friction to become isostatic in the rigid contact limit. The essential difference is that contact forces can no longer be regarded as enforcing hard geometric constraints like impenetrability, and hyperstatic configurations do not require exceptional arrangements or matching of particle sizes as in the frictionless case [32, 74, 76, 92].

Unlike us, Zhang and Makse [83] speculate that isostatic packings could be obtained in the limit of slow compressions of samples with ordinary values of $\mu$. This is however due to a divergence of interpretation, rather than a contradiction in numerical results, since their minimum coordination numbers $z^{*}$, excluding rattlers, are similar to ours, $z^{*} \simeq 4.5$. Zhang and Makse could only approach configurations devoid of hyperstaticity on setting the friction coefficient to infinity (see Section IV D 6 below). The degree of force indeterminacy per degree of freedom on the backbone is still equal, from (34), to 0.141 in $\mathrm{D}$ samples and 0.145 in $\mathrm{C}$ ones, and varies very little with compression rate in the range we explored, which extends to significantly smaller values than the ones used in [83], as stressed above. It is not obvious whether special experimental situations might occur in which real granular assemblies approach vanishing degrees of hyperstaticity.

\section{Distribution of normal forces}

Since the force-carrying structure maintains a nonvanishing degree of force indeterminacy even in the rigid limit for frictional packings, the force distribution in states B, C, and D, unlike in A configurations, is no longer a geometrically determined quantity in the rigid limit.

The distribution of normal components of contact forces (normalized by its average $\langle N\rangle$ ) is shown on Fig. 11 for all 4 configurations $\mathrm{A}, \mathrm{B}, \mathrm{C}$, and D, at the lowest pressure (as given in Table IV), at the end of the assembling process. We observe, as in many other numerical [25, 72, 93, 94] and experimental [95, 96] studies, an approximately exponential decay of $P(f)$ for large values, which is somewhat slower in states with low coordination number (in agreement with the values of $Z(2)$ given in Table IV]. It should be pointed out, however, that much larger differences between force distributions in the four studied states A, B, C, D will appear on increasing the confining pressure (see [37], paper II). This is already apparent in the dependence of $Z(2)$ on the previous history of D samples in table III.

All probability distribution functions show a local minimum for $f \rightarrow 0$, except in state C. Although it was remarked in past publications [97] that an upturn of the 


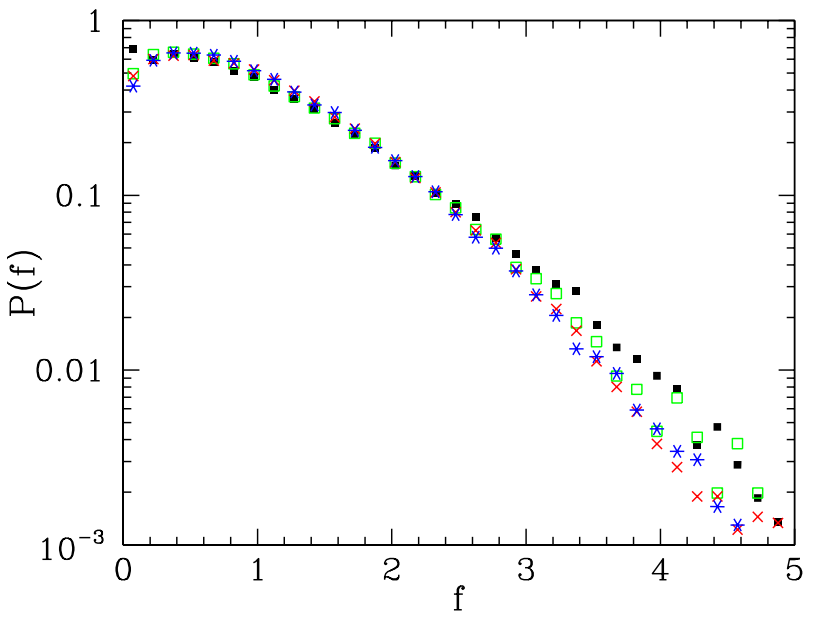

FIG. 11: (Color online) Distribution of normal forces, normalized by its average, $f=\frac{N}{\langle N\rangle}$, in states A (red crosses), B (blue asterisks), C (black square dots) and D (green open squares) at the lowest simulated pressure.

p.d.f. at low forces, as for $\mathrm{C}$ configurations, appeared when packings were not fully equilibrated, $\mathrm{C}$ configurations satisfy equilibrium requirements as well as the others.

\section{Friction mobilization}

As frictionless sphere packings are unstable for $z^{*}<$ 6 [32], a certain level of friction mobilization is required in states B to D, even under isotropic stresses. In particular, non-vanishing tangential forces are indispensable to ensure the equilibrium of grains with 2 and 3 contacts, for which they relate to the local geometric configuration, as discussed above (see e.g., Fig. 10). Some information about friction mobilization is given in Table VI. In Table VI we distinguish between contacts carrying normal forces larger and smaller than the average. Those two populations of contacts, as first distinguished in [97, 98], as the "strong" and "weak" networks, are attributed different roles, especially under anisotropic stresses. We merely use these categories here to gather information, in a compact, summarized form, about some aspects of force networks, which correlate to the force level. Table VI shows that, although three-coordinated particles tend to carry small forces on average, a notable proportion of them, and of the divalent ones, participate in the strong "force chains" with larger than average force levels. Friction mobilization is necessary in the contacts with such spheres, and reaches similar levels on average in the whole network. It it larger for contacts carrying small loads.

\section{The limit of large friction coefficients}

Motivated by the search for an isostatic limit in packings with intergranular friction, Zhang and Makse 83. assembled numerical packings with friction coefficients equal to infinity. Then they could get $z^{*} \simeq 4.15$. In order to investigate, in paper III [38] the elastic properties of tenuous contact networks, we also prepared a set of 5 configurations on compressing a granular gas under $P=1 \mathrm{kPa}$ with $\mu=+\infty$ and condition $I \leq 0.001$. These states, herafter referred to as $\mathrm{Z}$ configurations, have solid fraction $\Phi=0.5917 \pm 0.0008$, backbone coordination number $z^{*}=4.068 \pm 0.006$, proportion of rattlers $x_{0}=$ $(18.4 \pm 0.5) \%$ and of divalent beads $x_{2}=(6.8 \pm 0.5) \%$, and $\mathrm{H}$ is indeed small in that case, $\frac{\mathrm{H}}{N_{f}^{*}} \simeq 0.031$. It seems therefore very plausible that the degree of hyperstaticity vanishes in this case, as $\kappa \rightarrow \infty$, for slowly assembled packs. In the light of this observation, the absence of such a limit for finite $\mu$ can be attributed to sliding friction destabilizing barely rigid structures, which collapse and tend to form contacts in excess over the minimum count.

\section{E. Conclusions}

We summarize here the most salient results of Section IV about systems with friction.

\section{Diversity of states of equilibrated packings}

The variety of inner structures of isotropic bead packings we have obtained, as summed up in Table IV, shows that the solid fraction is not the only variable determining the internal state of an isotropic equilibrated packing. In particular, the backbone coordination number $z^{*}$ can vary throughout the whole interval from about 4.5 to 6 in the rigid limit $(\kappa \rightarrow+\infty)$, in packings with a solid fraction close to the RCP value. Some systems can be denser than others, but with a considerably smaller coordination number. Systems compacted by vibration should have smaller coordination numbers than systems assembled with low friction coefficients.

\section{Geometry and length scales}

Geometric characteristics corresponding to length scales above about 4 or $5 \%$ of the particle diameter correlate with density: this applies to the global shape and the area under the peaks of pair correlation function $g(r)$, to near neighbor gap-dependent coordination number $z(h)$, and to local neighbor arrangements around one bead, as measured by the local order parameters charted in Appendix D. 


\begin{tabular}{|c|ccccccccccc|}
\hline State & $X_{1}$ & $M_{1}$ & $M_{2}$ & $N^{(2)}$ & $X_{1}^{(2)}$ & $M_{1}^{(2)}$ & $M_{2}^{(2)}$ & $N^{(3)}$ & $X_{1}^{(3)}$ & $M_{1}^{(3)}$ & $M_{2}^{(3)}$ \\
\hline B & 0.42 & 0.016 & 0.018 & n. d. & n. d. & n. d. & n. d. & 0.23 & 0.09 & 0.02 & 0.01 \\
C & 0.41 & 0.14 & 0.18 & 0.58 & 0.19 & 0.14 & 0.17 & 0.24 & 0.09 & 0.13 & 0.04 \\
D & 0.41 & 0.16 & 0.22 & 0.71 & 0.22 & 0.16 & 0.18 & 0.26 & 0.10 & 0.15 & 0.05 \\
\hline
\end{tabular}

TABLE VI: For states B, C, and D, at the lowest pressure (see table IV]), proportion $X_{1}$ of contacts carrying normal forces larger than the average $\langle N\rangle$, average ratio $\frac{\|\mathbf{T}\|}{N}$ for contacts with $\left.N\right\rangle\langle N\rangle$ (respectively, $N<\langle N\rangle$ ) $M_{1}$ (resp. $M_{2}$ ). The same quantities with superscripts (2) and (3) apply to contacts implying spheres of coordination 2 and $3 . N^{(2)}, N^{(3)}$ are the average normal forces carried by these two populations of contacts, normalized by $\mathrm{Pa}^{2}$. Note that divalent grains are absent in state $\mathrm{B}$, and the corresponding quantities are therefore not defined.

Features associated with smaller scales, such as $z(h)$ for $h \leq 0.04 a$ or the shapes of the peaks of $g(r)-$ part of which approach a discontinuity in the rigid limit correlate with coordination number.

Finally, a third, smaller length scale $\kappa^{-1} a$ is associated with contact deflection and vanishes in the rigid limit. On this scale the geometric properties of the packing depend on the contact law.

\section{Role of rattlers}

Rattlers represent a significant fraction (above 10\%) of the particles in poorly coordinated systems, although the packings are stable. The treatment of rattlers - whether they are left floating in arbitrary positions (I) or gently pushed against the backbone by small forces (II) changes geometric data on the intermediate scale mentioned in the previous paragraph (Sec. IVE2), such as the exponent of a power-law fit of $z(h)$. Treatment II should be preferred if comparisons are to be made with packings under gravity. It leads to the definition of a "geometric" coordination number, $z^{I I}(0)$, different from the mechanical one $(z)$.

\section{Influence of micromechanical parameters}

Our data suggest (table III) that the states obtained on isotropically compressing a granular gas no longer depend on viscous damping parameter $\zeta$ in the limit of slow compression $(I \rightarrow 0)$. If the true value of the friction coefficient is used at this stage (i.e., without "lubrication"), the resulting state (our D configurations) can be regarded as a reference, loose packing limit of this assembling method. Other methods nevertheless result in lower densities.

\section{Force indeterminacy}

In samples assembled by isotropic compression, the backbone does not lose its force indeterminacy at low pressure, even in the slow compression limit, except for $\mu \rightarrow+\infty$. The degree of force indeterminacy, $\mathrm{H}$, decreases to about $14 \%$ of the number of backbone degrees of freedom in poorly coordinated systems with $\mu=0.3$ On computing $\mathrm{H}$ it is necessary to take into account the contribution of divalent grains, which define localized (harmless) floppy modes. Consequently the value of the backbone coordination number $z^{*}$ corresponding to $\mathrm{H}=0$ is slightly smaller than 4 .

\section{DISCUSSION AND PERSPECTIVES}

The most important novel feature of our simulation results is the wide variability of coordination numbers for the same density in isotropic packings. Most often, dense numerical samples are obtained on suppressing friction: A-type packings are simulated, in which the coordination number is high. C-type systems have about the same density, but a coordination number as low as in the loosest states $\mathrm{D}$ obtained by direct compression. In order to simulate dense laboratory samples, should one use A or C configurations ? The answer depends of course on how laboratory samples are assembled. Our results show that vibrated ones are likely to have smaller coordination numbers than lubricated ones for the same density. In paper III, we compare elastic properties of our B and C states to the ones measured by Jia and Mills [84] on glass bead samples either compacted by shaking the container or assembled with a lubricant.

In the X-ray tomography experiments by Aste et al. [6, 7], sphere packings are imaged with a resolution (voxel size) of about $4 \%$ of nominal diameter $a$, while the diameter distribution extends at least to $\pm 0.03 a$. In spite of serious efforts to eliminate the influence of size distribution by deconvoluting correlation data, their estimation of coordination numbers are well above the upper limit 6 in dense samples, which is in principle impossible under a low pressure. It seems that such experiments only provide access to the largest of the three length scales mentioned in Section IVE2 and are thus unable to distinguish between A-like and C-like microstructures. We shall see in paper III [38] that measurements of elastic moduli are much better suited to obtain information on coordination numbers by experimental means.

However, it is first necessary to assess the influence of the pressure level on the packing inner states. As hinted by the results of Table 【II, a quasistatic compression affects the force distribution and the level of friction mobi- 
lization. Elastic properties being usually measured above a certain confinement level (typically, a few tens of $\mathrm{kPa}$ ), the necessary study of the effects of a quasistatic compression is carried out in paper II [37].

Beyond the elastic properties, which characterize the response to small load increments, the quasistatic, elastoplastic mechanical behavior of packings prepared with different microstructures should also be studied. Peak deviator strength and dilatancy normally correlate with initial packing density [11, 12, 13, 21, 27]. But for one given density, what is the influence of the coordination number on the stress-strain curves ?

Granular systems are often packed under gravity by pouring samples in containers, and such processes, which do not necessarily produce homogeneous states 33 should be studied by numerical simulations too, and the analysis of dynamical effects in the assembling stage should be pursued. Adhesive contact forces, as in wet granular assemblies [82], can also greatly affect the preparation of solid granular packings [99]. Other perspectives to the present work are the investigation of the microstructure of polydisperse systems, and of assemblies of non-spherical particles [100].

\section{APPENDIX A: CONTACT ELASTICITY AND FRICTION}

The contact law between spherical elastic bodies, with a Coulomb criterion for friction applied locally (to the surface force densities), leads to complicated historydependent force-displacement relationships 39, 101]. Even in some cases with no slip anywhere in the contact region, the tangential stiffness $K_{T}$ of a contact was shown [102] to depend on the past history of the contact loading, and to change according to the direction of the displacement increment. Strictly speaking, the response of intergranular contacts, even to arbitrary small load increments, should not be termed "elastic". The simplified law we adopted involves a tangential stiffness $K_{T}$ depending on the normal deflection $h$, but independent of the current mobilization of friction. This is the same approximation as used in [29, 30]: the value of $K_{T}$ is the correct one in the absence of elastic relative tangential displacement, when $\mathbf{T}=0$.

However, as stressed in [40], such a model is thermodynamically inconsistent, for the elastic energy might increase at no cost. Consider, e.g., quasistatically reducing $h$ at constant $\delta \mathbf{u}_{T}$, thereby, according to this contact model, reducing normal force $N$ at constant $\mathbf{T}$, without reaching the Coulomb limit. The recoverable elastic energy stored in the contact is given by

$$
w=\frac{2}{5} \tilde{E} \sqrt{a} h^{5 / 2}+\frac{1}{2} \frac{\mathbf{T}^{2}}{K_{T}},
$$

which grows as $K_{T}$ decreases, without the external force doing any work, thus implying a net creation of energy. To avoid such effects, $\mathbf{T}$ is rescaled (as advocated by $\mathrm{O}$.
Walton [103]), whenever $N$ decreases to $N-\Delta N$, down to $\mathbf{T} \frac{K_{T}(N-\Delta N)}{K_{T}(N)}$, before accounting for tangential relative displacement increments. No such rule applies to increasing normal force cases. Such a procedure was shown by Elata and Berryman [40] to systematically produce energy dissipation in cyclic loadings of the contact.

Such peculiarities of the contact law affect the form and, in fact, the very definition of an elastic response of the contact network, an issue which will be discussed in paper III.

\section{APPENDIX B: TRANSPORT OF CONTACT FORCES DUE TO PARTICLE MOTION}

In molecular dynamics calculations, as well as in static approaches (as outlined in Section $\amalg$ (IC) one has to relate small contact force increments in any contact to the small displacements $\mathbf{u}_{i}$ and rotations $\Delta \theta_{i}$ of the grains.

Increments $\Delta\left(N_{i j} \mathbf{n}_{\mathbf{i j}}\right)$ and $\Delta \mathbf{T}_{i j}$ of the normal and tangential parts of the force in the contact between grains $i$ and $j$ have two different origins: they stem from the contact law, as written down in Section II] and also from the motion of the particle pair. As the grains move, so does the deformed contact region, and therefore the resulting contact force changes. The relevant formulae are derived and written below for small increments in the static case. For dynamical computations, displacements are to be replaced by velocities, and increments by time derivatives.

The normal force variation is simply

$$
\Delta\left(N_{i j} \mathbf{n}_{i j}\right)=\Delta N_{i j} \mathbf{n}_{i j}+N_{i j} \Delta \mathbf{n}_{i j}
$$

with

$$
\Delta \mathbf{n}_{i j}=\frac{1}{\left\|\mathbf{r}_{i j}\right\|}\left(\underline{\underline{\mathbf{1}}}-\mathbf{n}_{i j} \otimes \mathbf{n}_{i j}\right) \cdot\left(\tilde{\mathbf{u}}_{j}-\tilde{\mathbf{u}}_{i}-\underline{\underline{\epsilon}} \cdot \mathbf{r}_{i j}\right),
$$

while $\Delta N_{i j}$ is related by the Hertz law to the variation in the normal deflection of the contact.

For the tangential reaction we introduce the decomposition

$$
\Delta \mathbf{T}_{i j}=\Delta \mathbf{T}_{i j}^{(1)}+\Delta \mathbf{T}_{i j}^{(2)}
$$

Increments with superscript (1) are associated, via the contact law, to the relative displacement of the contact point, which defines the constitutive part of the stiffness matrix discussed in Section IIC, and superscript (2) labels increments of kinematic origin. We assume that the magnitude of the contact force is unchanged in the absence of relative displacement at the contact $\left(\delta \mathbf{u}_{i j}=0\right)$, and thus we write

$$
\Delta \mathbf{T}_{i j}^{(2)}=\Delta \theta_{i j} \times \mathbf{T}_{i j}
$$

$\Delta \theta_{i j}$ denoting the rotation of the contact region. $\Delta \theta_{i j}$ can be split in a rolling part $\Delta \theta_{i j}^{(R)}$, orthogonal to $\mathbf{n}_{i j}$, 
and a pivoting one, $\Delta \theta_{i j}^{(P)}$, along $\mathbf{n}_{i j} \cdot \Delta \theta_{i j}^{(R)}$ is determined by the incremental change in $\mathbf{n}_{i j}$ :

$$
\Delta \theta_{i j}^{(R)}=\mathbf{n}_{i j} \times \Delta \mathbf{n}_{i j}
$$

As to the pivoting part $\Delta \theta_{i j}^{(P)}$, it is natural to equate it to the average rotation of the two particles around the normal direction:

$$
\Delta \theta_{i j}^{(P)}=\frac{1}{2} \mathbf{n}_{i j} \cdot\left(\Delta \theta_{i}+\Delta \theta_{j}\right) \mathbf{n}_{i j}
$$

This choice is such that the rotation of the contact force coincides with the rotation of the pair in contact if both objects move together like one rigid body (a condition of objectivity [49]).

Injecting (B2) into Eqns. (B4) and (B5), one readily obtains the appropriate formula for $\Delta \mathbf{T}_{i j}^{(2)}$,

$$
\begin{aligned}
\Delta \mathbf{T}_{i j}^{(2)}= & -\left[\mathbf{T}_{i j} \cdot\left(\tilde{\mathbf{u}}_{j}-\tilde{\mathbf{u}}_{i}-\underline{\underline{\epsilon}} \cdot \mathbf{r}_{i j}\right)\right] \frac{\mathbf{n}_{i j}}{r_{i j}} \\
& +\frac{1}{2}\left[\left(\Delta \theta_{i}+\Delta \theta_{j}\right) \cdot \mathbf{n}_{i j}\right]\left(\mathbf{n}_{i j} \times \mathbf{T}_{i j}\right) .
\end{aligned}
$$

With the notations of Section IIC, the contribution of contact $i, j$ to the $3 \times 3$ diagonal block of $\underline{\underline{\mathbf{K}}}^{(2)}$ which expresses the dependence of $\mathbf{F}_{i}^{\text {ext }}$ on displacement $\mathbf{u}_{i}$ is the non-symmetric tensor

$$
-\frac{N_{i j}}{r_{i j}}\left(\underline{\underline{\mathbf{1}}}-\mathbf{n}_{i j} \otimes \mathbf{n}_{i j}\right)+\frac{\mathbf{n}_{i j} \otimes \mathbf{T}_{i j}}{r_{i j}} .
$$

In general, the geometric stiffness matrix $\underline{\mathbf{K}}^{(2)}$ is thus not symmetric, except in frictionless sphere packings, which are analogous to central force networks. We also note that terms of order $N / R$ or $\|T\| / R$ in $\underline{\underline{K}}^{(2)}$ correspond to terms of order $K_{N}$ or $K_{T}$ in $\underline{\underline{K}}^{(1)}$, which are always very much larger.

In the frictionless case, $\underline{\mathbf{K}}^{(2)}$ is a symmetric, negative matrix if forces are repulsive, as discussed by Alexander [91]. Any mechanism on the backbone leads to an instability: the potential energy of the externally applied load is strictly decreasing in that motion. This destabilizing effect can also be directly established in the rigid case, as shown in 32]. This is the reason why stable packings of frictionless spheres in equilibrium under some externally applied load are devoid of mechanisms involving the backbone.

In general stiffness matrices were discussed by Kuhn and Chang [49], and by Bagi [50]. Those authors gave general results for $\underline{\mathbf{K}}^{(2)}$ with particles of arbitrary shapes, which coincide with ours in the case of spherical balls.

\section{APPENDIX C: STRESS-CONTROLLED MOLECULAR DYNAMICS}

It might be noted that the original Parrinello-Rahman equations slightly differ from ours. First, Eqn. (6) is writ- ten down with an additional term

$$
m_{i} \ddot{s}_{i}^{(\alpha)}=\frac{1}{L_{\alpha}} F_{i}^{(\alpha)}-2 \frac{\dot{L}_{\alpha}}{L_{\alpha}} m_{i} \dot{s}_{i}^{(\alpha)} .
$$

Then, eqn. (7) is written with a different stress tensor, $\underline{\underline{\pi}}$, the definition of which involves a particular reference

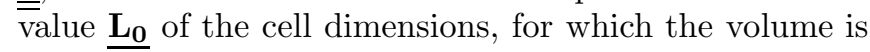
$\Omega_{0} \cdot \underline{\underline{\underline{i}}}$ is related to the Cauchy stress tensor $\underline{\underline{\Sigma}}$ by

$$
\underline{\underline{\pi}}=\frac{\Omega}{\Omega_{0}} \underline{\underline{L}}_{0} \cdot \underline{\underline{\mathbf{L}}}^{-1} \cdot \underline{\underline{\underline{\Sigma}}} \cdot{ }^{\mathbf{T}} \underline{\underline{\mathbf{L}}}^{-1} \cdot{ }^{\mathbf{T}} \underline{\underline{L}}_{0}^{-1}
$$

$\underline{\pi}$ is a symmetric tensor known as the second Piola$\overline{\bar{K}}$ irchhoff stress tensor [104 (also called thermodynamic tension by some authors [105, 106]). Tensor $\underline{\underline{\pi}}$ can be used to express the power $\mathcal{P} / \Omega_{0}$ of internal forces, per unit volume in the reference (undeformed) configuration.

The metric tensor $\underline{\underline{\mathcal{G}}}={ }^{\mathbf{T}} \underline{\underline{\mathbf{L}}}_{0}^{-1} \cdot{ }^{\mathbf{T}} \underline{\underline{\mathbf{L}}} \cdot \underline{\underline{\mathbf{L}}} \cdot \underline{\underline{\mathbf{L}}}_{0}^{-1}$ expresses the distance between current points as a function of their coordinates in the reference configuration. The difference between $\underline{\underline{\mathcal{G}}}$ and the unit tensor defines the GreenLagrange strain tensor [104], e

$$
\underline{\underline{\mathbf{e}}}=-\frac{1}{2}(\underline{\underline{\mathcal{G}}}-\underline{\underline{\mathbf{I}}}) \text {. }
$$

Then $\stackrel{\pi}{=}$ is such that, for whatever strain history

$$
\mathcal{P}=\Omega_{0} \underline{\underline{\pi}}: \underline{\underline{\dot{e}}}
$$

If the last term of Eqn. (17) is replaced by $\frac{\left(L_{0}^{\alpha}\right)^{2}}{\Omega_{0} L_{\alpha}}$, and if (C1) is used instead of (6), then, in the case when interparticle forces derive from a potential $V$, function of particle positions $\mathbf{r}_{i}$ and orientations, the system of equations conserves the total energy

$$
\begin{aligned}
H & =\frac{1}{2} \sum_{i} m_{i} \dot{\mathbf{s}}_{i} \cdot{ }^{\mathbf{T}} \underline{\underline{\mathbf{L}}} \cdot \underline{\underline{\mathbf{L}}} \cdot \dot{\mathbf{s}}_{i}+\frac{1}{2} \sum_{i} I_{i} \omega_{i}^{2}+V \\
& +\frac{1}{2} M \cdot{ }^{\mathbf{T}} \underline{\underline{\mathbf{L}}}: \underline{\underline{\mathbf{L}}}+V+\frac{\Omega_{0}}{2} \underline{\underline{G}}: \underline{\pi} .
\end{aligned}
$$

Such equations would tend to impose a constant PiolaKirchhoff stress.

Granular assemblies are however dissipative systems, and energy conservation is not a crucial issue as in molecular systems. In practice, we observed that omission of the extra term of (C1) as well as control of Cauchy, rather than Piola-Kirchhoff stresses, did not affect the approach to equilibrium configurations. Yet, on considering small motions and elastic properties close to an equilibrium configuration, one may prefer dealing with external forces deriving from a potential. We note that this is indeed the case if we use Eqns. (C1) and (7) with an isotropic $\underline{\underline{\boldsymbol{\Sigma}}}$, $\underline{\underline{\boldsymbol{\Sigma}}}=P \underline{\underline{\mathbf{1}}}$, in which case the external stress control is asso-

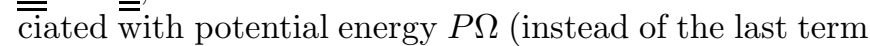
in Eqn. C5. 


\section{APPENDIX D: COMPARISON OF LOCAL BOND ORDER PARAMETERS WITH EXPERIMENTAL OBSERVATIONS}

Although the samples were not isotropic, owing to the role of gravity in the preparation stage, and not perfectly homogeneous, because of lateral walls, the X-ray tomography experiments by Aste et al. 7] provided an unprecedented wealth of results on the geometry of sphere packings. The local arrangement of neighbors around one bead were classified according to the values taken by the pair $\left(Q_{4}^{\text {loc }}(i), Q_{6}^{\text {loc }}(i)\right.$, for different choices of the distance $d_{c}$ that defines the bonds. For each sample and choice of $d_{c}$, the proportion of spheres having the most frequent range of values $\left(\hat{Q}_{4} \pm 0.05, \hat{Q}_{6} \pm 0.05\right)$ corresponding to some typical disordered arrangement was recorded, as well as the frequency of occurrence of values $(0.191 \pm 0.05,0.574 \pm 0.05)$ and $(0.097 \pm 0.05,0.485 \pm 0.05)$ respectively corresponding to fcc-like and hcp-like local ordering. Those values are compared to the ones we observed in numerical samples of similar density in tables VII and VIII (we kept the same $\left(\hat{Q}_{4}, \hat{Q}_{6}\right)$ couple).

TABLE VII: Most frequently values $\left(\hat{Q}_{4}(i), \hat{Q}_{6}(i)\right)$ observed in the measurements of [7] in a dense sample $(\Phi=0.640 \pm 0.005$, called sample $\mathrm{F}$ in [7]), and fraction (dis.) of local configurations within interval $\left(\hat{Q}_{4} \pm 0.05, \hat{Q}_{6} \pm 0.05\right)$ obtained in experiments and in numerical simulations with similar densities : $\mathrm{A}, \mathrm{A}^{\prime}$ and $\mathrm{C}$.

\begin{tabular}{cccccc}
\hline \hline Sample & $d_{c}$ & $\left(\hat{Q}_{4}, \hat{Q}_{6}\right)$ & dis $(\%)$ & $f c c(\%)$ & hcp $(\%)$ \\
\hline Aste et al. & 1.1 & $(0.23,0.44)$ & 31 & 6 & 4 \\
dense & 1.2 & $(016,0.45)$ & 38 & 4 & 12 \\
$\Phi \simeq 0.640$ & 1.3 & $(0.13,0.42)$ & 43 & 1 & 17 \\
$(\mathrm{~F}$ in $[7])$ & 1.4 & $(0.10,0.38)$ & 47 & 3 & 13 \\
\hline A & 1.1 & (same & 32 & $<10^{-2}$ & 4 \\
A & 1.2 & values & 37 & $<10^{-2}$ & 5 \\
A & 1.3 & as & 43 & $<10^{-2}$ & 8 \\
A & 1.4 & above $)$ & 46 & $<10^{-2}$ & 12 \\
\hline A $^{\prime}$ & 1.1 & (same & 31 & $<10^{-2}$ & 5 \\
A $^{\prime}$ & 1.2 & values & 40 & $<10^{-2}$ & 6 \\
A $^{\prime}$ & 1.3 & as & 45 & $<10^{-2}$ & 10 \\
$\mathrm{~A}^{\prime}$ & 1.4 & above $)$ & 48 & $<10^{-2}$ & 15 \\
\hline C & 1.1 & (same & 31 & $<10^{-2}$ & 4 \\
C & 1.2 & values & 37 & $<10^{-2}$ & 5 \\
C & 1.3 & as & 43 & $<10^{-2}$ & 8 \\
C & 1.4 & above) & 46 & $<10^{-2}$ & 13 \\
\hline \hline
\end{tabular}

The fraction of beads with the typical disordered configuration of neighbors (marked dis. in the tables) are very close in numerical packings and in the experimental one of the same densities, and the frequency of occurrence of hcp-like local environments also compares well, although it does not seem to share the same dependence on the threshold distance $d_{c}$ defining bonds. However, the small fraction of fcc-like beads observed in the laboratory is
TABLE VIII: Same as table VII for experimental samples of lower densities, one with $\Phi=0.626 \pm 0.008$ (called sample $\mathrm{D}$ in [7]), another with $\Phi=0.596 \pm 0.006$ (called sample B in [7]), to which values obtained in simulated samples B and $\mathrm{D}$, of similar densities, are respectively compared.

\begin{tabular}{cccccc}
\hline \hline Sample & $d_{c}$ & $\left(\hat{Q}_{4}, \hat{Q}_{6}\right)$ & dis $(\%)$ & $f c c(\%)$ & $h c p(\%)$ \\
\hline Aste et al. & 1.1 & $(0.25,0.44)$ & 28 & 4 & 1 \\
& 1.2 & $(0.19,0.44)$ & 35 & 2 & 7 \\
$\Phi \simeq 0.626$ & 1.3 & $(0.15,0.40)$ & 42 & 1 & 11 \\
(D in [7]) & 1.4 & $(0.11,0.36)$ & 46 & 1 & 8 \\
\hline B & 1.1 & (same & 30 & $<10^{-2}$ & 2.7 \\
B & 1.2 & values & 38 & $<10^{-2}$ & 7.6 \\
B & 1.3 & as & 43 & $<10^{-2}$ & 10 \\
B & 1.4 & above $)$ & 46 & $<10^{-2}$ & 10 \\
\hline \hline Aste et al. & 1.1 & $(0.30,0.45)$ & 24 & 3 & 1 \\
loose & 1.2 & $(0.23,0.44)$ & 32 & 2 & 3 \\
$\Phi \simeq 0.596$ & 1.3 & $(0.16,0.38)$ & 37 & 1 & 5 \\
(B in [7]) & 1.4 & $(0.14,0.35)$ & 43 & 2 & 5 \\
\hline D & 1.1 & (same & 24 & $<10^{-2}$ & 1.0 \\
D & 1.2 & values & 32 & $<10^{-2}$ & 4.4 \\
D & 1.3 & as & 37 & $<10^{-2}$ & 6.1 \\
D & 1.4 & above $)$ & 43 & $<10^{-2}$ & 7.5 \\
\hline \hline
\end{tabular}

absent in the simulations. Many cirmcumstances can be invoked to explain these differences, including of course the different packing history of the numerical and experimental samples, which in the the latter case involves gravity and anisotropy. It can be remarked once again that A' samples are a little more ordered than A ones (with slightly larger fractions of hcp-like local configurations), from which $\mathrm{C}$ samples are quite indistinguishable, as the quantities measured here do not depend on whether pairs of neighbors are actually in contact.

\section{APPENDIX E: ANALYSIS OF THE FREE MOTION OF DIVALENT SPHERES}

We first give here the appropriate formulae to describe the free motion of divalent grains, then report on numerical stability tests.

The equations are specialized to the case of equal-sized spheres of dimater $a$, as in all the simulations reported in the present paper. Let $i$ denote the label of the divalent grain in contact with its neighbors labelled $j$ and $k$. The line joining the centers of $j$ and $k$ is parallel to unit vector e, defined as

$$
\mathbf{e}=\frac{\mathbf{n}_{i k}-\mathbf{n}_{i j}}{\left\|\mathbf{n}_{i k}-\mathbf{n}_{i j}\right\|},
$$

and the distance $D$ of the center of $i$ to this line is

$$
D=a \sqrt{1-\left(\mathbf{e} \cdot \mathbf{n}_{i j}\right)^{2}}
$$


$\omega_{0}$ denoting the angular velocity of the center of $i$ about this axis, the translational velocity of $i$, in its free motion depicted on Fig. 10, will be

$$
\mathbf{v}_{i}=\omega_{0} D \mathbf{t},
$$

the unit vector $\mathbf{t}$ being orthogonal to the plane containing the centers of $i, j$, and $k$ :

$$
\mathbf{t}=\frac{\mathbf{n}_{i j} \times \mathbf{n}_{i k}}{\left\|\mathbf{n}_{i k} \times \mathbf{n}_{i j}\right\|} .
$$

Its angular velocity will be

$$
\boldsymbol{\Omega}_{i}=2 \omega_{0} \mathbf{e} .
$$

With such a choice, the instantaneous velocity of the (material) contact points between $i$ and $j$, or between $i$ and $k$ satisfy:

$$
\mathbf{v}_{i}+\boldsymbol{\Omega}_{i} \times \frac{a}{2} \mathbf{n}_{i j}=\mathbf{v}_{i}+\boldsymbol{\Omega}_{i} \times \frac{a}{2} \mathbf{n}_{i k}=0,
$$

as requested in a relative motion which is a combination of rolling and pivoting.

It is easy to check that the rules defined in Appendix B ensure that the tangential components of the contacts $i, j$ and $i, k$ rotate with the contact points around the axis joining the centers of $k$ and $j$ with angular velocity $\omega_{0}$. In other words, the geometric stiffness matrix $\underline{\underline{\mathbf{K}}}^{(2)}$ does not determine whether the mechanism associated with a two-coordinated bead is stable.

We check for stability with numerical means, as follows. Starting from an equilibrium configuration, we first choose the potentially dangerous mechanisms, those involving relatively large contact forces, of the order of the average normal force or even larger. Thus, grains $j$ and $k$ undergo significant changes in the moment of the contact force with the mobile grain $i$. Then one such mobile divalent bead is attributed a velocity and a angular velocity according to Eqns. E1 and E2, with a value of $\omega_{0}$ small enough for the centrifugal acceleration to be negligible (equal to $10^{-4}|| \mathbf{F}_{i j}||$ ). The evolution of the whole packing under constant stress is then simulated with MD. Such numerical experiments were performed with the most fragile packings, D samples under low confining pressures. In all cases studied, as expected, the motion of the mechanism entails very slow changes in the configuration, if any. The mobile grain maintains a constant angular velocity while nearly exactly following its circular trajectory for a long time, with hardly any change in kinetic energy. These calculations are rather slow and costly, and we therefore limited our investigations to 10 tests for 2 pressure levels in the $\mathrm{D}$ series, $P=1 \mathrm{kPa}$ and $P=10 \mathrm{kPa}$. At the lowest pressure $P=1 \mathrm{kPa}$, corresponding to $\kappa \simeq 181000$, these motions were often observed to lead to a small rearrangement of the packing, in which kinetic energy spreads over all degrees of freedom, a significant fraction of the contacts, up to $25 \%$, go through a sliding stage, the contact network is slightly modified and the system restabilizes in a slightly different configuration, with a small density increase (typically of order $10^{-5}$ ). In other cases, the freely moving grain stops when it collides with a third grain other than the two with which it is maintaining contact. The system then finds a new equilibrium configuration without rearranging, only a few contacts temporarily reach a sliding status. On repeating similar tests at a larger confining pressure, $P=10 \mathrm{kPa}$ (still close to the rigid limit), the occurrence of this second scenario became much more frequent than the first, which was never observed in the 10 tests performed.

The difference between stable and unstable cases is better appreciated on redoing the tests with a modified MD calculation method, in which only the initially existing contacts are taken into account. Thus one only investigates the properties of the pre-existing contact network. If it breaks, the system globally falls apart, and nearly all contacts in the packing eventually open. This is the unstable case. In the stable case the mobile particle can turn several times around the line of centers of its two contacting neighbors without notable changes in kinetic energy and the contact network is maintained. This behavior is illustrated on Fig. 12, which displays trajectories of mobile grains in the plane orthogonal to e. Note that such a procedure reveal instabilities that are prevented by the appearance of a third contact of the mobile grain, and hence overestimates the frequency of occurrence of unstable configurations. 8 out of 10 such tests led to an instability in $\mathrm{D}$ samples under $1 \mathrm{kPa}$. This proportion fell to 2 out of 10 under $10 \mathrm{kPa}$.

Two possible conclusions may be drawn. On the one hand we may deem the $\mathrm{D}$ configurations under low pressures imperfectly stabilized, as some free motions might eventually cause configurational changes. Or, since anyway the evolution is so slow, it may be pointed out, on the other hand, that the slightest amount of dissipation in rolling would stop the free motion. In realistic systems the velocity a a free rolling motion always decays in time.

Since the obtention of stable, equilibrated states in which all divalent grains have been made threecoordinated would be computationnally very costly, and as the occurrence of small instabilities related to such mechanisms appear to decrease fastly under growing confinement, we adopted the second attitude and regarded $\mathrm{D}$ and $\mathrm{C}$ configurations with a few percent of divalent particles as acceptable equilibrium states.
[1] H. J. Herrmann, J.-P. Hovi, and S. Luding, eds., Physics of Dry Granular Media (Balkema, Dordrecht, 1998).
[2] H. Hinrichsen and D. E. Wolf, eds., The Physics of 


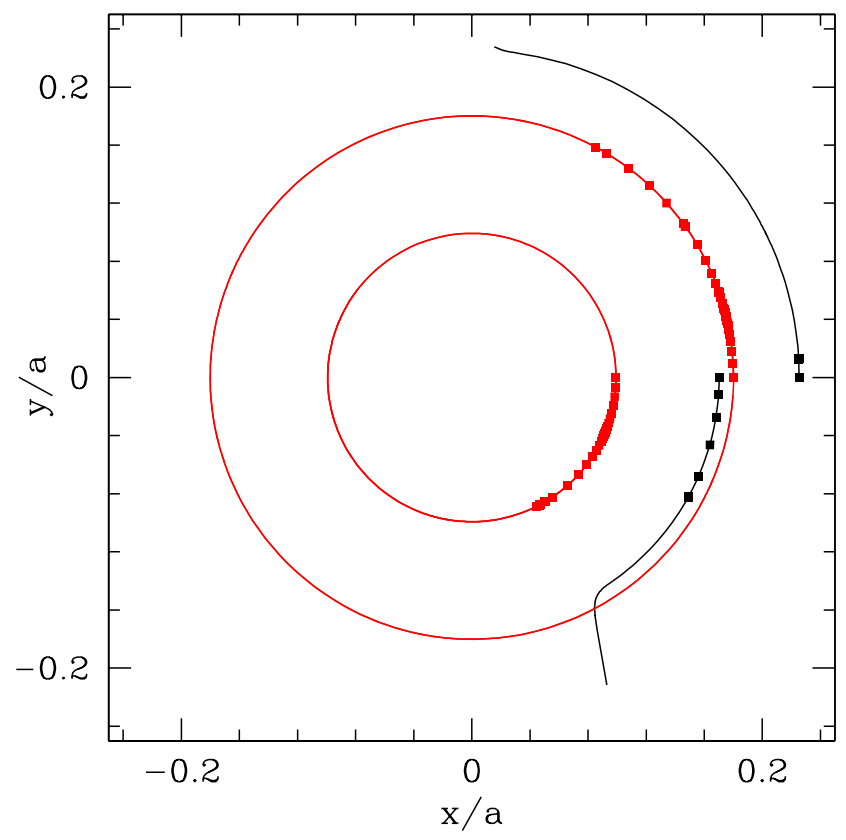

FIG. 12: (Color online) Examples of trajectories of mobile divalent grains in the plane orthogonal to vector e. All trajectories begin at $x=D, y=0$. Continuous lines depict the results obtained on ignoring contact creation, which end up, in 2 cases out of the 4 represented, in instabilities (black lines), while complete circles are observed in the other, stable ones (red). Dots mark the same trajectories, which are in practice arrested by other contacts when contact creation is taken into account.

Granular Media (Wiley-VCH, Berlin, 2004).

[3] R. García Rojo, H. J. Herrmann, and S. McNamara, eds., Powders and Grains 2005 (Balkema, Leiden, 2005).

[4] A. J. Liu and S. R. Nagel, eds., Jamming and rheology (Taylor \& Francis, New York, 2001).

[5] P. Richard, P. Philippe, F. Barbe, S. Bourlès, X. Thibault, and D. Bideau, PRE 68, 020301(R) (2003).

[6] T. Aste, M. Saadatfar, A. Sakellariou, and T. J. Senden, Physica A 339, 16 (2004).

[7] T. Aste, M. Saadatfar, and T. J. Senden, PRE 71, 061302 (2005).

[8] D. Cumberland and R. Crawford, The Packing of Particles (Elsevier, Amsterdam, 1987).

[9] D. Bideau and J. Dodds, eds., Physics of Granular Media (Nova Science Publishers, 1991).

[10] S. Torquato, T. M. Truskett, and P. G. Debenedetti, Physical Review Letters 84, 2064 (2000).

[11] D. M. Wood, Soil Behaviour and Critical State Soil Mechanics (Cambridge University Press, 1990).

[12] J. Biarez and P.-Y. Hicher, Elementary Mechanics of Soil Behaviour (A. A. Balkema, Rotterdam, 1993).

[13] J. K. Mitchell, Fundamentals of soil behavior (Wiley, New York, 1993).

[14] M. Oda, Soils and Foundations 12, 17 (1972).

[15] J. R. F. Arthur and B. K. Menzies, Géotechnique 22, 115 (1972).

[16] S. Fukushima and F. Tatsuoka, Soils and Foundations
24, 40 (1984).

[17] L. Vanel, D. Howell, D. Clark, R. P. Behringer, and E. Clément, Phys. Rev. E 60, R5040 (1999).

[18] P. A. Cundall and O. D. L. Strack, Géotechnique 29, 47 (1979).

[19] R. J. Bathurst and L. Rothenburg, Mechanics of Materials 9, 65 (1990).

[20] F. Radjai and S. Roux, in 107], pp. 21-24.

[21] F. Radjai and S. Roux, in [2], pp. 165-187.

[22] L. Rothenburg and N. P. Kruyt, International Journal of Solids and Structures 41, 5763 (2004).

[23] J.-N. Roux and F. Chevoir, Bulletin des Laboratoires des Ponts et Chaussées 254, 109 (2005).

[24] L. E. Silbert, D. Ertaş, G. S. Grest, T. C. Halsey, and D. Levine, Physical Review E 65, 031304 (2002).

[25] L. E. Silbert, G. S. Grest, and J. W. Landry, Physical Review E 66, 061303 (2002).

[26] H. Makse, D. Johnson, and L. Schwartz, Physical Review Letters 84, 4160 (2000).

[27] C. Thornton, Géotechnique 50, 43 (2000).

[28] A. S. J. Suiker and N. A. Fleck, ASME Journal of Applied Mechanics 71, 350 (2004).

[29] H. Makse, N. Gland, D. Johnson, and L. Schwartz, Physical Review Letters 83, 5070 (1999).

[30] H. A. Makse, N. Gland, D. L. Johnson, and L. Schwartz, Physical Review E 70, 061302 (2004).

[31] C. O'Hern, L. E. Silbert, A. J. Liu, and S. R. Nagel, Physical Review E 68, 011306 (2003).

[32] J.-N. Roux, Physical Review E 61, 6802 (2000).

[33] N. S. Rad and M. T. Tumay, ASTM Journal of Geotechnical Testing 10, 31 (1987).

[34] N. Benahmed, J. Canou, and J.-C. Dupla, ComptesRendus Académie des Sciences, Mécanique 332, 887 (2004).

[35] E. R. Nowak, J. B. Knight, E. Ben-Naim, H. M. Jaeger, and S. R. Nagel, Physical Review E 57, 1971 (1998).

[36] P. Philippe and D. Bideau, Europhysics Letters 60, 677 (2002).

[37] I. Agnolin and J.-N. Roux, Internal states of model isotropic granular packings: compression and pressure cycles, first companion paper, second in the series (2007).

[38] I. Agnolin and J.-N. Roux, Internal states of model isotropic granular packings: elastic properties, second companion paper, third in the series (2007).

[39] K. L. Johnson, Contact Mechanics (Cambridge University Press, 1985).

[40] D. Elata and J. G. Berryman, Mechanics of Materials 24, 229 (1996).

[41] E. Somfai, J.-N. Roux, J. Snoeijer, M. van Hecke, and W. van Saarloos, PRE 72, 021301 (2005).

[42] M. Parrinello and A. Rahman, Journal of Applied Physics 52, 7182 (1981).

[43] J. Christoffersen, M. M. Mehrabadi, and S. NematNasser, Journal of Applied Mechanics 48, 339 (1981).

[44] J.-P. Hansen and I. R. McDonald, Theory of Simple Liquids (Academic Press, 1986).

[45] P. Cundall, in Micromechanics of Granular Materials, edited by M. Satake and J. T. Jenkins (Elsevier, Amsterdam, 1988), pp. 113-123.

[46] B. Servatius and H. Servatius, in 51], pp. 1-19.

[47] S. McNamara, R. García Rojo, and H. J. Herrmann, Physical Review E 72, 021304 (2005).

[48] S. McNamara and H. J. Herrmann, Physical Review E 
74, $061303(2006)$.

[49] M. R. Kuhn and C. S. Chang, International Journal of Solids and Structures 43, 6026 (2006).

[50] K. Bagi, Granular Matter 9, 109 (2007).

[51] M. F. Thorpe and P. M. Duxbury, eds., Rigidity Theory and Applications, Fundamental Materials Research (Kluwer Academic, 1998).

[52] A. Donev, S. Torquato, and F. H. Stillinger, PRE 71, 011105 (2005).

[53] G. Combe and J.-N. Roux, in Deformation characteristics of geomaterials, edited by $\mathrm{H}$. di Benedetto, T. Doanh, H. Geoffroy, and C. Sauzéat (Swets and Zeitlinger, Lisse, 2003), pp. 1071-1078.

[54] F. Tatsuoka, M. Sakamoto, T. Kawamura, and S. Fukushima, Soils and Foundations 26, 65 (1986).

[55] G. Reydellet and E. Clément, Physical Review Letters 86, 3308 (2001).

[56] G. Combe, Mécanique des matériaux graulaires et origines microscopiques de la déformation (Presses du Laboratoire Central des Ponts et Chaussées, Paris, 2002).

[57] GdR MIDI, European Physical Journal E 14, 341 (2004).

[58] F. da Cruz, S. Emam, M. Prochnow, J.-N. Roux, and F. Chevoir, Physical Review E 72, 021309 (2005).

[59] O. Pouliquen, C. Cassar, Y. Forterre, P. Jop, and M. Nicolas, in 3], pp. 859-865.

[60] P. Jop, Y. Forterre, and O. Pouliquen, Journal of Fluid Mechanics 541, 167 (2005).

[61] P. Jop, Y. Forterre, and O. Pouliquen, Nature 44, 727 (2006).

[62] B. J. Alder and T. E. Wainwright, J. Chem. Phys. 31, 459 (1959).

[63] B. D. Lubachevsky and F. H. Stillinger, Journal of Statistical Physics 60, 561 (1990).

[64] B. D. Lubachevsky, F. H. Stillinger, and E. N. Pinson, Journal of Statistical Physics 64, 501 (1991).

[65] A. Donev, S. Torquato, F. H. Stillinger, and R. Connelly, Journal of Computational Physics 197, 139 (2004).

[66] I. Volkov, M. Cieplak, J. Koplik, and J. R. Banavar, Physical Review E 66, 061401 (2002).

[67] S. Emam, J.-N. Roux, J. Canou, A. Corfdir, and J.-C. Dupla, in [3], pp. 49-52.

[68] N. Benahmed, Ph.D. thesis, École Nationale des Ponts et Chaussées, Champs-sur-Marne, France (2001).

[69] V. Luchnikov, A. Gervois, P. Richard, L. Oger, and J.P. Troadec, Journal of Molecular Liquids 96-97, 185 (2002).

[70] R. Connelly, Structural Topology 14, 43 (1988).

[71] A. R. Kansal, S. Torquato, and F. H. Stillinger, Physical Review E 66, 041109 (2002).

[72] S. Ouaguenouni and J.-N. Roux, Europhysics Letters, 39, 117 (1997).

[73] J.-N. Roux, in Proceedings of the Saint-Venant Symposium on Multiple Scale Analysis and Coupled Physical Systems (Presses de l'Ecole Nationale des Ponts et Chaussées, Paris, 1997), pp. 577-584.

[74] C. F. Moukarzel, Physical Review Letters 81, 1634 (1998).

[75] C. F. Moukarzel, in [51], pp. 125-142.

[76] A. Tkachenko and T. A. Witten, Physical Review E 60,
627 (1999).

[77] C. F. Moukarzel, Granular Matter 3, 41 (2001).

[78] C. F. Moukarzel, in [2], pp. 23-43.

[79] L. E. Silbert, A. J. Liu, and S. R. Nagel, Physical Review E 73, 041304 (2006).

[80] Détermination des masses volumiques minimales et maximales des sols non cohérents, French Standard NFP 94-059 (1992).

[81] G. Y. Onoda and E. G. Liniger, Physical Review Letters 64, 2727 (1990).

[82] M. M. Kohonen, D. Geromichalos, M. Scheel, C. Schier, and S. Herminghaus, Physica A 39, 7 (2004).

[83] H. P. Zhang and H. A. Makse, Physical Review E 72, 011301 (2005).

[84] X. Jia and P. Mills, in [107], pp. 105-112.

[85] G. T. Nolan and P. E. Kavanagh, Powder Technology 72, 149 (1992).

[86] W. M. Visscher and M. Bolsterli, Nature 239, 504 (1972).

[87] R. Jullien and P. Meakin, Europhysics Letters 4(12), 1385 (1987).

[88] G. C. Barker and A. Mehta, Physical Review A 45, 3435 (1992).

[89] A. Mehta, G. C. Barker, and J. M. Luck, Journal of Statistical Mechanics: Theory and Experiment p. P10014 (2004).

[90] I. Bratberg, F. Radjai, and A. Hansen, Physical Review E 66, 031303 (2002).

[91] S. Alexander, Phys. Rep. 296, 65 (1998).

[92] S. Ouaguenouni and J.-N. Roux, in [108], pp. 188-191.

[93] F. Radjai, M. Jean, J.-J. Moreau, and S. Roux, Phys. Rev. Lett. 27, 274 (1996).

[94] S. J. Antony, Phys. Rev. E 63, 011302 (2000).

[95] D. M. Mueth, H. M. Jaeger, and S. R. Nagel, Phys. Rev. E 57, 3164 (1998).

[96] D. L. Blair, N. W. Mueggenburg, A. H. Marshall, H. Jaeger, and S. R. Nagel, Physical Review E 63, 041304 (2001).

[97] F. Radjai, D. Wolf, S. Roux, M. Jean, and J.-J. Moreau, in [108], pp. 169-179.

[98] F. Radjai, D. E. Wolf, M. Jean, and J.-J. Moreau, Physical Review Letters 80, 61 (1998).

[99] F. A. Gilabert, J.-N. Roux, and A. Castellanos, Phys. Rev. E 75, 011303 (2007).

[100] A. Donev, R. Connelly, F. H. Stillinger, and S. Torquato, PRE 75, 051304 (2007).

[101] R. D. Mindlin and H. Deresiewicz, ASME Journal of Applied Mechanics 20, 327 (1953).

[102] D. L. Johnson and A. N. Norris, Journal of Mechanics and Physics of Solids 45, 1025 (1997).

[103] O. Walton, private communication.

[104] J. Salençon, Handbook of continuum mechanics. General concepts, Thermoelasticity. (Springer, 2001).

[105] J. R. Ray and A. Rahman, JCP 76, 4423 (1984).

[106] A. Lemaitre and C. Maloney, Journal of Statistical Physics 123, 415 (2006).

[107] Y. Kishino, ed., Powders and Grains 2001 (Swets \& Zeitlinger, Lisse, 2001).

[108] D. Wolf and P. Grassberger, eds., Friction, Arching, Contact Dynamics (World Scientific, Singapore, 1997). 